DINÂMICA de CONFLITOS POPULACIONAIS

Nelson A. Chaves Ramirez

DISSERTAÇÃO APRESENTADA

$\mathrm{AO}$

INSTITUTO DE MATEMÁTICA E ESTATÍSTICA

DA

UNIVERSIDADE DE SÃO PAULO

PARA OBTENÇÃO DO GRAU

DE

MESTRE EM MATEMÁTICA APLICADA

Área de Concentração: Matemática Aplicada

Orientador: Prof. Dr. Sergio Muniz Oliva

Durante a elaboração deste trabalho,

o autor recebeu apoio financeiro do CNPQ

-São Paulo, Julio de 1998- 


\title{
Dinâmica de Conflitos Populacionais
}

\author{
Este exemplar corresponde à redação \\ final da dissertação devidamente \\ corregida e defendida por Nelson A. Chaves Ramirez \\ e aprovada pela comissão julgadora.
}

São Paulo, 10 de Julio de 1998.

Banca examinadora:

- Prof. Dr. Sergio Muniz Oliva (orientador) - IME-USP

- Prof. Dr. Alexandre Nolasco Carvalho - ICMC-USP

- Prof. Dr. Hildebrando Munhoz Rodrigues - ICMC -USP 
A Fabiana 


\title{
Resumo
}

Consideramos um modelo dinâmico para jogos evolutivos com difusão e introduzimos um termo que regulará o fluxo pela fronteira. Provamos, usando técnicas de semi-grupo e análise não-linear, quando uma solução de equilíbrio, que é uma estratégia evolucionária estável (E.S.S.), é instável ou estável com respeito a perturbações espaciais. Tal resultado generaliza os encontrados na literatura, mais expecificamente os resultados de Vickers et al. (1993).

\begin{abstract}
We consider a dynamical model for evolutionary games with diffusion and introduce a boundary term that will control the flux through the boundary. We prove, using semi-groups and non-linear analysis techniques, when an equilibrium solution, which is an evolutionary stable strategy (E.S.S.), is unstable or stable under spatial perturbations. Such results generalize the results found in the literature, particulary the ones found in Vickers et al. (1993).
\end{abstract}




\section{Agradecementos}

Es muy grande el número de personas que hicieron posible que yo llegase hasta aqui y seria imposible nombrarlas a todas. Entre ellas, existen algunas que tuvieron y tienen participacion decisiva. Primero que nada quiero agradecer a mi esposa a quien le dedico este trabajo; que siempre me acompaño en los momentos mas duros. A mis padres, que siempre me estimularon a seguir adelante y que muchas veces dejaron de disfrutar de la vida por darnos hasta lo imposible. A Nestor Charquero, Jubenal Porrini, Gustavo Alzugaray, a la Hna. Redentor, a Tubino, quienes tuvieron una incidencia fundamental en mi formacion. También quiero agradecer a Ani Pizzuti.

A mi grandes amigos de la infancia Horacio Kouss y a Pablo Delepiani; a la persona increible que es Julio Sabatino, a J. Kalemkerian, S.Gianoni, G.Brida, G. Groisman, M. Lanzzilota, a F. Abadie, a la turma de la Vila Gomes, Marcia, Carolina y especialmente a Juan Egaña. A Lucia quien me ayudó muchisimo con el portugués.

Como dije antes algunas tuvieron incidencia fundamental, por eso quisiera agradescer muy especialmente a mi orientador Sergio Muniz Oliva, quien confio en mi, mas que yo mismo, con mis idas y venidas, sin el cual este trabajo hubiese sido imposible.

Quisiera agradecer también a F. Pelaes, a A. Abella, A Pereyra, A Robella y a R. Arocena quienes me ayudaron mucho durante mis estudios de Licenciatura, a Alfredo Jones y a Flavio Cohelo quienes me impulsaron junto con Sergio a venir a São Paulo "a qual a pesar de seu tamanho tambem tem seus encanto". 


\section{Ajedrez II}

Tenue rey, sesgo alfil, encarnizada reina, torre directa y peón ladino sobre lo negro y blanco del camino buscan y libran su batalla armada.

No saben que la mano señalada del jugador gobierna su destino, no saben que un rigor adamantino sujetan su albedrío y su jornada.

También el jugador es prisionero (la sentencia es de Omar) de otro tablero de negras noches y blancos días.

Dios mueve al jugador, y éste, la piesa.

¿que dios detrás de Dios la trama empieza de polvo y tiempo y sueño y agonias?

Jorge Luis Borges 


\section{Sumário}

0.1 o modelo biológico . . . . . . . . . . . . . . 3

0.2 Objetivos deste trabalho . . . . . . . . . . . . 7

0.3 Formulação matemática do modelo . . . . . . . . 7

1 Preliminares 9

1.1 Operadores Dissipativos e Semigrupos . . . . . . . . . . 9

1.2 Inversão da transformada de Laplace. . . . . . . . . . . . . 13

1.3 Operadores Setoriais, Potências Fracionárias e Semigrupos Analíticos. . . . . . . . . . . . . . . 16

1.4 Expoente Fracionário de um Operador. . . . . . . . . . . 20

1.5 Exemplos . . . . . . . . . . . . . . 22

2 O Problema de Cauchy 23

2.1 Existência local e unicidade . . . . . . . . . . . . 24

2.2 Estabilidade e instabilidade dada pela primeira aproximação. 26

2.3 Exemplo. . . . . . . . . . . . . . . 27

3 Atratores e Conjuntos Invariantes para $C^{r}$-semigrupos 28

4 Atratores para Problemas Parabólicos com Condição de Fronteira não Linear 31

4.1 Introdução. . . . . . . . . . . . . . . . . . . . 31

4.2 Espaços de Lebesgue . . . . . . . . . . . . . . . . . . . . . . . . . . . . 35

4.3 Teoria de Interpolação e Domínios . . . . . . . . . . . . . . 35

4.4 Potência fracionária negativa de um espaço. . . . . . . . . . . 37

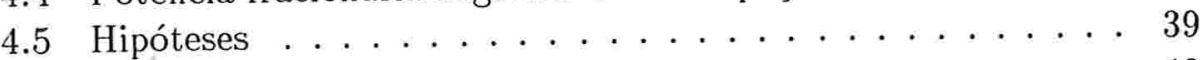

4.6 Existência local . . . . . . . . . . . . . . . . . 40

4.7 Resultados de Regularidade . . . . . . . . . . . . . . . . . . . 42

4.8 Limitação do Semigrupo . . . . . . . . . . . . . . . . . . . . . . . . . 43

4.9 Existência de Atratores Globais. . . . . . . . . . . . 45 
5 Linearização e Estabilidade $\quad 47$

5.1 A Diferencial de Frechet de uma Função . . . . . . . . . . 47

6 Existência do Atrator Global para O Problema Biológico 52

6.1 Verificação das Hipóteses . . . . . . . . . . . 54

6.1.1 Testando as Hipóteses para a não Linearidade no Interior 54

6.1.2 Construção da não Linearidade na Fronteira . . . . . . 54

6.1.3 Teste da interação entre a não linearidade da fronteira

e do interior . . . . . . . . . . . . . 56

6.2 Aplicação dos Resultados Abstratos . . . . . . . . . . 57

6.2.1 Limitação do Semigrupo, Sub e Super-solução . . . . . 57

6.3 Existência do Atrator Global . . . . . . . . . . . . 58

7 Equilíbrios 59

7.1 Equilíbrio Instável . . . . . . . . . . . . . 59

7.1.1 Primeiro caso com fronteira Neumann . . . . . . 61

7.1.2 Segundo caso com controle pela fronteira . . . . . . 63

A Autovalores e Parâmetros $\quad 66$ 


\section{Introdução}

\subsection{O modelo biológico}

Nós consideramos uma população na qual os indivíduos tomam parte em disputas, sempre aos pares. Assumiremos que tais disputas são simétricas, isto é, não existem diferenças óbvias entre os participantes. A situação anterior foi considerada por Maynard Smith [20].

Cada indivíduo sempre joga uma de $r$ diferentes estratégias, etiquetadas de 1 a $r$, e se o $i$-ésimo joga com o $j$-ésimo jogador, este recebe um pagamento ("pay-off") $\left(a_{i j}\right)$.

Estes ganhos afetam as reproduções seguintes, que seguem sempre a mesma estratégia. Logo temos uma matriz $r \times r$ de premios, $A=\left(a_{i j}\right)$. O estado na qual se encontra a população pode ser descrito pelo vetor de probabilidade $p=\left(p_{i}\right)$, onde $p_{i}$ é a proporção da população que joga a estratégia $i$.

O ganho esperado que obterá o $i$-ésimo jogador é

$$
E(i, p)=\sum_{j=1}^{r} a_{i j} p_{j}=(A p)_{i} .
$$

Também definamos $E(q, p)=\sum_{i=1}^{r} q_{i} E(i, p)$, portanto $E(q, p)$ é o pagamento esperado pelo grupo de indivíduos na população quando uma proporção $q_{i}$ joga a estratégia $i$.

Definição 0.1.1 Uma população é estável se o vetor $p$ satisfaz as seguintes condições:

$$
E(p, q) \geq E(q, p)
$$


Se $q \neq p$ e se verifica a igualdade então,

$$
E(p, q)>E(q, q) \forall q
$$

Definição 0.1.2 Se o anterior se verifica então $p$ é dito uma estratégia estável de evolução (E.S.S).

As noções anteriores são estáticas e não dão indicações de como um E.S.S pode ser escolhido ou quão rápido os imigrantes podem ser expulsos. Taylor e Jonker [25] sugerem a seguinte dinâmica:

$$
\frac{d p_{i}}{d t}=p_{i}\left((A p)_{i}-p^{t} A p\right) \quad 1 \leq i \leq r
$$

que permite investigar o desenvolvimento da população no tempo. Zeemann [30] sugere buscar os pontos de equilíbrio estáveis (locais) ao invés dos E.S.S..

Estas idéias são muito úteis mas não suficientes. Isto é, imigração e mutação, que estão no coração do problema, envolvem variações espaciais assim como temporais.

Em geral a inclusão de termos espaciais não pode se fazer automaticamente. Se todos os membros da população se dispersam simultaneamente, o modelo seria:

$$
\frac{\partial p_{i}}{\partial t}=p_{i}\left[(A p)_{i}-p^{t} A p\right]+D_{i} \Delta p_{i} \quad 1 \leq i \leq r
$$

Isto foi proposto por Hadeler [10] em populações genéticas que é uma extensão do modelo proposto por Fisher [7]. Contudo, é razoável esperar que em muitas situações os coeficientes de dispersão sejam afetados pela estratégia que vem sendo seguida. Este caso é um problema com a normalização, e qualquer modelo introduzirá novas suposições e variáveis. Nós usaremos um conjunto de equações introduzidas por Vickers [27]. A forma funcional foi escolhida para assegurar a conservação total da densidade da população, estamos designando por $v_{i}(x, t)$ a densidade dos $i$ - estrategistas na posição $x$ no tempo $t$ e por $V(x, t)$ a densidade total, as equações são:

$$
\frac{\partial v_{i}}{\partial t}=f_{i}(v)+d_{i} \Delta v_{i} \quad 1 \leq i \leq r
$$

onde

$$
f_{i}(v)=v_{i}\left(\frac{(A v) i}{V}-\frac{v^{t} A v}{V^{2}}\right)
$$


e $V=\sum_{i=1}^{r} v_{i}$. Aqui $d_{i}$ são os coeficientes de dispersão dos i estrategistas e a equação se verifica em uma região $\Omega \subset R^{l}$ a qual é limitada e tem fronteira suave $\partial \Omega, v$ satisfaz condição Neumann homogênea em $\partial \Omega$.

A forma especial de $f$, que é clássica nestes modelos, é coerente com a do modelo proposto por Taylor-Jonker [25].

É uma álgebra simples verificar as seguintes propriedades:

$$
\begin{gathered}
f(k v)=k f(v) \\
\sum_{i=0}^{r} f_{i}(v)=0
\end{gathered}
$$

Como conseqüência de (4), se $v$ é solução de (3) também o é $k v$, para todo $k$. Esta falta de unicidade reflete o fato que no correspondente modelo de reação, os $v_{i}$ são frequências, portanto a condição $\sum v_{i}=1$ é imposta. Para nosso sistema de reação difusão não serão mais frequências o que causará dificuldades técnicas quando trabalhamos com soluções estacionárias, por conseguinte, será necessário impor uma condição integral para solucionar a unicidade. Segue da equação (4) junto com a equação (1) e integrando em $\Omega$, que $\int_{\Omega} V(x, t) d x$ é constante. Em outras palavras, o total de indivíduos não muda em $\Omega$ (a qual é uma condição que substitui parcialmente a condição anterior, imposta sobre as frequências).

Turing mostrou que "patterns" espaciais estão associados com equilíbrios que são estáveis com respeito a pertubações homogêneas mas instáveis com respeito as heterogêneas, tendo em vista os diferentes tipos de estabilidade, Vickers [29] introduz a seguinte terminologia.

Definição 0.1.3 Diremos que um ponto de equilibrio espacialmente constante $\bar{v}$ é temporalmente estável se é assintoticamente estável em relação a perturbações homogêneas do sistema de reação difusão, isto é, com todos os $d_{i}=0$.

$O$ ponto de equilíbrio $\bar{v}$ é dito espacialmente estável com um sistema particular de coeficientes de perturbação $d_{i}$, se e somente se é assintoticamente estável por pertubaçóes espaciais heterogêneas, e uniformemente estável se $o$ anterior se verifica para todos os $d_{i} \geq 0$.

Suponhamos que $\bar{v}$ é um ponto de equilíbrio espacialmente constante do sistema de reação (3) e ponhamos $V=\sum \bar{v}_{i}$. Consideremos primeiro a situação na qual todos os $v_{i}$ são constantes em espaço. Então a equação (3) se simplifica a :

$$
\frac{d p_{i}}{d t}=p_{i}\left((A p)_{i}-p^{t} A p\right) \quad 1 \leq i \leq r
$$


onde $p_{i}=\frac{v_{i}}{V}$ é um vetor de probabilidade dependendo só do tempo. Assumiremos que a equação (1) tem um ponto do equilíbrio assintoticamente estável $\bar{m}=\frac{\bar{v}}{\bar{V}}\left(m_{i}>0\right)$, e investigamos sob que circunstâncias é assintoticamente estável para (3). Em geral, a estabilidade de qualquer solução dependerá em parte da forma, assim como do tamanho da região $\Omega$, pois somente certas pertubações são possíveis. Seja $\rho$ um autovetor de $-\Delta$, em $\Omega$, isto é, existe uma função não nula $\phi$ tal que

$$
-\Delta \phi=\rho \phi \quad x \in \Omega
$$

e

$$
\frac{\partial \phi}{\partial n}=0
$$

Existe um número infinitos de tais autovalores: quando $\Omega=(0, \pi)$, temos $\rho=0,1,4,9, \ldots$

A estabilidade da solução $\bar{v}$ foi investigada supondo uma solução da forma

$$
v(x, t)=\bar{v}+a \exp (w t+i k x) .
$$

A condição de que uma solução não trivial existe (ver Vickers [27]) é que

$$
\operatorname{det}\left[m_{i} a_{i j}-m_{i}\left(m^{t} A\right)_{j}-\left(d_{i} \rho+w\right) \delta_{i j}\right]=0 .
$$

Como $\rho=0$ é sempre um autovalor do $\Delta$, esta equação tem uma solução degenerada $w=0$ correspondente às perturbações que são constantes no espaço e no tempo. Esta raiz é irrelevante quando queremos decidir sobre a estabilidade. Portanto $\bar{v}$ é assintoticamente estável se todas as outras raízes da equação tem parte real negativa, para todos os autovalores $\rho$.

Supondo que $m$ é um ponto de equilíbrio da equação (1), assintoticamente estável, mas $m$ não é um E.S.S.. Então existe um vetor de probabilidade $q$ para o qual

$$
q^{t} A q \geq m^{t} A q
$$

isto é, $m$ é invadível por $q$. O caso mais fácil a investigar é quando $q$ é uma estratégia pura a qual no seguinte teorema escolhemos o vetor $(1,0,0 \ldots \ldots .$.$) .$

Teorema 0.1.1 (Vickers et al. [29]) Seja $m$ um ponto de equilibrio temporalmente estável do sistema (1) para o qual $m_{i} \geq 0$ para todo $i$. Se

$$
a_{11} \geq\left(m^{t} A\right)_{1}
$$

então existe um conjunto de coeficientes de dispersão $d_{i} \geq 0$, para todo $i$ tal que $m$ é um ponto assintoticamente instável da equação (3), isto é se uma estratégia pura pode invadir $m$ então não é uniformemente espacialmente estável. 
Com estas considerações, usando as técnicas de D.Henry [11], provamos no capítulo 5 , sem fazer uso da solução, os teoremas (2.1), (2.2) de Vickers et all. [29].

\subsection{Objetivos deste trabalho}

Pretendemos neste trabalho estabelecer uma formulação matemática sólida para este modelo, de tal forma que esta formulação nos possibilite:

i) Provar o teorema 0.1 .1 , sem se utilizar a forma especial (7), que aumentará os domínios permitidos;

ii) Mostrar que podemos modificar, de forma controlada, o fluxo pela fronteira e obter os mesmos resultados obtidos por Vickers, sem impor que a estratégia seja ganhadora ou não para (1);

iii) Mostrar que, mesmo introduzindo um fluxo pela fronteira, o modelo, do ponto de vista matemático, está "bem posto" (ou seja, temos existência e unicidade) e pode ser um modelo biologico coerente, isto é, não é "instável" (mostraremos a existência de um atrator global).

Gostaríamos de resaltar que estes resultados são originais e, a nosso ver, dão outra dimensão ao modelo.

\subsection{Formulação matemática do modelo}

Para atingir os objetivos mencionados anteriormente, prosseguiremos como no estudo de modelos de química, biologia e ecologia que envolvem problemas de reação-difusão em um domínio $\Omega$ com condição de fronteira Neumann linear ou não. Mais precisamente iremos escrever nosso modelo na forma

$$
\left\{\begin{array}{c}
u_{t}=\operatorname{Div}(a \nabla u)+\sum_{j=1}^{n} B_{j}(x) \frac{\partial u}{\partial x_{j}}+\lambda u-f(u) \quad \text { em } \Omega \\
\frac{\partial v_{i}}{\partial n_{\alpha}}=g(u) \quad \text { em } \partial \Omega
\end{array}\right.
$$

onde $u=\left(u_{1}, \ldots, u_{N}\right), N \leq 1, a(x)=\operatorname{diag}\left(a_{1}(x), \ldots, a_{N}(x)\right), a_{i} \in C^{\mathbf{1}}(\Omega)$, $0<a_{i}(x) x \in \Omega, \lambda>0$ e $f, g$ são funções de $R^{N}$ em $R^{N}$.

A literatura deste tipo de sistema é extensa, dentre os inúmeros resultados encontrados, gostaríamos de citar que Pao [14] estabeleceu uma relação entre $f$ e $g$ tal que o sistema final era "estável". Mais tarde, Alikakos [1], seguindo as ideias de Friedman [8], mostrou existência global e algum comportamento assintótico das soluções. Trabalhando com domínios unidimencionais, Henry [12] estabeleceu uma relação precisa entre $f$ e $g$ para que o sistema final seja Morse-Smale. Seguindo paralelamente, Amann, [2], [3] 
estabeleceu um procedimento para colocar (9) em uma formulação abstrata e assim estabeleceu existência e unicidade.

Combinando os resultados de Amann [3] e Henry [12], Carvalho, Oliva, Pereira e Rodriguez Bernal [6] e Oliva e Pereira [18] estabeleceram uma condição sobre $f$ e $g$ para que o sistema seja estável.

Neste trabalho seguiremos esta formulação e utilizaremos os resultados de Henry [11] para estabelecer a estabilidade ou instabilidade dos pontos críticos e para mostrar que, mesmo que o ponto crítico seja instável, que o sistema possui sempre um atrator global.

O trabalho seguirá da seguinte forma:

Capítulo 1 Estabelecemos a nomenclatura e os resultados básicos, necessários para colocar o problema (9) numa forma abstrata. Mais precisamente faremos um apanhado geral sobre a teoria de semigrupos, baseando-se no trabalho de Henry [11], e Pazy [15].

Capítulo 2 Seguindo o trabalho de Henry [11] estabelecemos a existência e unicidade das soluções do problema abstrato e estabelecemos os resultados de estabilidade e instabilidade dos pontos de equilíbrio.

Capítulo 3 Seguindo o trabalho de Hale [9], resumiremos o ferramental necessário para a existência de atratores globais.

Capítulo 4 Seguindo o trabalho de Amann, [2], [3] e Oliva e Pereira [18], estabelecemos os resultados gerais para os sistemas do tipo (9).

Capítulo 5,6 e 7 Aplicamos as técnicas estabelecidas nos capítulos anteriores ao nosso modelo, cumprindo os objetivos mencionados anteriormente. 


\section{Capítulo 1}

\section{Preliminares}

\subsection{Operadores Dissipativos e Semigrupos}

Nesta seção apresentamos os resultados básicos de semigrupos fortemente contínuos e uniformemente contínuos, seus resolventes e suas representações integrais, que podem ser encotrados por exemplo em [15].

O objetivo é aplicar os resultados da teoria de semigrupos para se obter informações sobre as soluções de equações diferenciais parciais. Como ilustração comecemos com um simples exemplo. Consideramos o problema de Cauchy.

$$
\frac{\partial u}{\partial t}=\frac{\partial u}{\partial x} ; \quad x>0, t>0, u(x, 0)=g(x) .
$$

Onde $u(x, t)$ é uma função de duas variáveis $x$ e $t$. Podemos fixar $t$ e definir a correspondente função denotada por, digamos $u_{t}$ definida por

$$
u_{t}(x)=u(t, x)
$$

Isto gera uma família a um parâmetro, de funções $\left(u_{t}\right)_{t \geq 0}$. Logo para cada $t \geq 0$ associamos a apropriada função $u_{t}$ da família, isto é

$$
u(t)=u_{t} .
$$

Então $u$ aplica $[0, \infty)$ sobre um espaço de funções, que pode ser escolhido dentro de vários, por exemplo podemos escolher $L^{p}$ com $1 \leq p<\infty$. Isto nos leva a utilizar ferramentas de cálculo em espaços de Banach, e podemos interpretar a equação

$$
\frac{\partial u}{\partial t}=\frac{\partial u}{\partial x}
$$


da seguinte forma. No lado direito fixemos $t$, podemos considerar isto como $A u_{t}$ onde $A$ é o operação diferenciação (definido da maneira usual) aplicado a uma função fixa $u_{t}$. Podemos interpretar o lado esquerdo como a derivada com respeito ao parâmetro $t$ da aplicação $u:[0, \infty) \rightarrow X$ onde $X$ é um espaço de Banach apropriado. Como $u$ é uma função de uma só varíavel, $t$, escrevemos $\frac{\partial u}{\partial t}$ como sendo $\frac{d u}{d t}$. Estas considerações nos levam ao estudo das equações diferenciais em espaços de Banach $X$ da forma

$$
\frac{d u}{d t}=A u(t)
$$

onde $A: D(A) \in X \rightarrow X$ é um operador (relacionado com a equação diferencial parcial em questão) e temos como solução

$$
u:[0, \infty) \rightarrow X
$$

Para generalizar e deixar precisas as considerações anteriores precisamos de alguns resultados e definições.

Definição 1.1.1 Sejam $X$ um espaço de Banach e $L: D(L) \subset X \rightarrow X$ um operador linear fechado. O conjunto resolvente de $L, \rho(L)$, é o conjunto de todos os número complexos $\lambda$ para os quais $\lambda I-L$ é inversível, isto é, o operador resolvente

$$
R(\lambda: L)=(\lambda I-L)^{-1}
$$

é um operador limitado em $X$.

O conjunto $\sigma(L)=C \backslash \rho(L)$ é chamado o espectro de $L$.

Definição 1.1.2 Um $C^{0}$ - semigrupo em um espaço de Banach $X$ é uma família de operadores lineares contínuos em $X, T(t), t \geq 0$, satisfazendo

i) $T(0)=I$

ii) $T(s+t)=T(s) \cdot T(t)$ para todo $s, t \geq 0$

iii) $T(t) x \rightarrow x$ quando $t \rightarrow 0^{+}$para cada $x \in X$.

Definição 1.1.3 Um semigrupo é uniformemente contínuo se

i) $T(0)=I$

ii) $T(s+t)=T(s) \cdot T(t)$ para todo $s, t \geq 0$

$e$

$$
\lim _{t \rightarrow 0}\|T(t)-I\|_{\mathcal{L}(X)}=0
$$


Definição 1.1.4 O gerador infinitesimal $L$ de um $C^{0}$-semigrupo $T(t)$, $t \geq 0$ é definido por:

$$
L x=\lim _{t \rightarrow 0} \frac{1}{t}(T(t) x-x)
$$

sendo seu domínio formado por todos os pontos de $X$ para os quais este limite existe. Usualmente, escrevemos $T(t)=e^{L t}$.

Definição 1.1.5 Seja $T(t), t \geq 0 u m C^{0}$ - semigrupo definido num espaço de Banach $X$. Dizemos que $T(t)$ é um semigrupo analítico em $X$ se, para cada $x \in X, t \rightarrow T(t) x$ é uma aplicação analítica para $0 \leq t<\infty$.

Definição 1.1.6 Sejam $R^{+}=[0, \infty)$ e X um espaço métrico completo. Uma família de funções $T(t): X \rightarrow X, t \geq 0$ é um $C^{0}$ - semigrupo não linear se:

i) $T(0)=I$

ii $) T(t+s)=T(t) T(s), t \geq 0, s \geq 0$

iii) $T(t) x$ é contínua em $(t, x) \in R^{+} \times X$.

Se $X$ é um espaço de Banach, $T(t): X \rightarrow X, t \geq 0$, satisfaz i), ii), iii) $e$ além disto,

iv) $T(t) x$ possui derivadas de Frechet em relação a $x$ contínuas até ordem $r, r \geq 1$ para $(t, x) \in R^{+} \times X$,

então $T(t): X \rightarrow X, t \geq 0$, é um $C^{r}-$ semigrupo não linear.

Teorema 1.1.1 Seja A um operador linear, então A é o gerador infinitesimal de um semigrupo uniformemente contínuo se, e somente se, A é um operador linear limitado.

Teorema 1.1.2 Sejam $T(t)$ e $S(t)$ dois semigrupos unifomemente contínuos. Se $\lim _{t \rightarrow 0} \frac{T(t)-I}{t}=A=\lim _{t \rightarrow 0} \frac{S(t)-I}{t}$ então $S(t)=T(t)$.

Os teoremas anteriores são importantes pois caracterizam os geradores infinitesimais dos semigrupos uniformemente contínuos. Mas os operadores provenientes de equações diferenciais parciais não são limitados, logo nos restringimos ao estudo de $C^{0}$-semigrupos.

Teorema 1.1.3 Seja $T(t)$ um $C^{0}$-semigrupo. Então existem constantes $\omega \geq 0$ e $M \geq 0$ tais que

$$
\|T(t)\| \leq M e^{\omega t}
$$

Estas estimativas são extremamente importantes pois fornecem o comportamento assintótico do semigrupo. 
Teorema 1.1.4 Seja $T(t)$ um $C^{0}$-semigrupo e seja A seu gerador infinitesimal. Então

i)Para $x \in X$, temos

$$
\lim _{h \rightarrow 0} \frac{1}{h} \int_{t}^{t+h} T(s) x d x=T(t) x
$$

ii)Para $x \in X$, temos

$$
\int_{0}^{t} T(s) x d s \in D(A) \quad e \quad A \int_{0}^{t} T(s) x d s=T(t) x-x
$$

iii) $x \in D(A)$, temos $T(t) x \in D(A) e$

$$
\frac{d T(t) x}{d t}=A T(t) x=T(t) A x
$$

iv)Para $x \in D(A)$, temos

$$
T(t) x-T(s) x=\int_{s}^{t} T(\tau) A x d \tau=\int_{s}^{t} A T(\tau) x d \tau
$$

$\mathrm{O}$ teorema anterior torna precisa a relação entre $C^{0}$-semigrupo e geradores infinitesimais com as equações diferenciais em espaços de Banach, ou seja o gerador caracteriza a equação e $T(t)$ fornece a solução.

Teorema 1.1.5 (Pazy [15]) (Hille-Yosida) Seja A um operador linear de $X$ em $X$. Então A é o gerador infinitesimal de um $C^{0}$-semigrupo satisfazendo $\|T(t)\| \leq M e^{\omega t}$ se e somente se

i) A é fechado e $\overline{D(A)}=X$;

ii) $O$ conjunto resolvente de A contém a semireta $(\omega, \infty) e$

$$
\left\|R(\lambda: A)^{n}\right\| \leq \frac{M}{(\lambda-\omega)^{n}}, \lambda>\omega, \quad n=1,2, \ldots
$$

Observação 1.1.1 A condição de que todo real $\lambda \geq \omega$ está no conjunto resolvente de $A$, conjunto com (1.1), implica que cada número complexo $\lambda$ satisfazendo $\operatorname{Re} \lambda \geq \omega$ se encontra em $\rho(A) e$

$$
\left\|R(\lambda: A)^{n}\right\| \leq \frac{M}{(\operatorname{Re} \lambda-\omega)^{n}}
$$

para $\operatorname{Re} \lambda \geq \omega, n=1,2 \ldots$. 
Uma aplicação do teorema de Hille-Yosida é a seguinte. Seja $\Omega \in R^{n}$ um aberto cuja fronteira denotaremos por $\Gamma$. Denotamos por

$$
Q=\Omega \times] 0, \infty[\text { e } \Sigma=\Gamma \times] 0, \infty[,
$$

isto é, $\Sigma$ é a fronteira lateral do cilindro $Q$. Consideramos o problema seguinte. Achar uma função $u(x, t): \bar{\Omega} \times[0, \infty[\rightarrow R$ tal que

$$
\left\{\begin{array}{c}
\frac{\partial u}{\partial t}=\Delta u \text { em } Q \\
u=0 \text { em } \Sigma \\
u(x, 0)=u_{0} \text { em } \Omega
\end{array}\right.
$$

Onde $t$ é normalmente denominado tempo e $u_{0}(x)$ é uma função dada. Se definimos o operador não limitado $A: D(A) \rightarrow X$ por

$$
\left\{\begin{array}{c}
u \in D(A)=H^{2}(\Omega) \cap H_{0}^{1}(\Omega) \\
A u=-\Delta u
\end{array}\right.
$$

Se comprova, aplicando o teorama de Hille Yosida (ver [4]), que a equação do calor (1.3) tem uma única solução.

\subsection{Inversão da transformada de Laplace.}

Um dos problemas da teoria de semigrupos de operadores é a relação entre o semigrupo e seu gerador infinitesimal.

Dado o semigrupo $T(t)$, podemos obter o gerador infinitesimal, através de sua definição, como:

$$
A x=\lim _{t \rightarrow 0} \frac{T(t) x-x}{t}, \quad x \in D(A) .
$$

Uma forma diferente de se caracterizar A é através de seu resolvente, para isto podemos utilizar a observação (1.1.1). Esta observação nos diz que, se $\|T(t)\| \leq M e^{\omega t}$, então

$$
R(\lambda: A) x=\int_{0}^{\infty} e^{-\lambda t} T(t) x d t \quad x \in X, \quad \operatorname{Re} \lambda>\omega .
$$

Do ponto de vista das equações diferenciais, é mais interessante se obter $T(t)$ de seu gerador infinitesimal. A razão para isso é que, para $x \in$ $D(A), T(t) x$ é a solução do problema inicial

$$
\frac{d u}{d t}-A u=0 \quad u(0)=x .
$$


que é desconhecida e $A$ define a equação, que é conhecida. Esta seção está dedicada ao problema de representar $T(t)$ a partir de seu gerador infinitesimal. Se $T(t)$ satisfaz $\|T(t)\| \leq M e^{\omega t}$ então o resolvente do $A$ satisfaz (1.4), isto é, o resolvente de $A$ é a transformada de Laplace do semigrupo associado. Portanto, esperamos obter o semigrupo a partir do resolvente de $A$, invertendo a transformada de Laplace. O primeiro passo é estabelecer o seguinte lema.

Lema 1.2.1 Seja B um operador linear limitado. Se $\gamma>\|B\|$ então

$$
e^{t B}=\frac{1}{2 \pi i} \int_{\gamma-i \infty}^{\gamma+i \infty} e^{\lambda t} R(\lambda: B) d \lambda=\frac{1}{2 \pi i} \lim _{x \rightarrow \infty} \int_{\gamma-i x}^{\gamma+i x} e^{\lambda t} R(\lambda: B) d \lambda
$$

A convergência é na topologia uniforme dos operadores em $t$ em conjuntos limitados.

Com isto podemos estabelecer o seguinte teorema, que resolverá o problema.

Teorema 1.2.1 Seja $B$ o gerador infinitesimal de um $C^{0}$-semigrupo $T(t)$ satisfazendo $\|T(t)\| \leq M e^{\omega t}$ e seja $\gamma>\max (0, \omega)$. Se $x \in D(A)$ então

$$
\int_{0}^{t} T(s) x d s=\frac{1}{2 \pi i} \int_{\gamma-i \infty}^{\gamma+i \infty} e^{\lambda t} R(\lambda: B) x \frac{d \lambda}{\lambda}
$$

Uma conseqüência importante é o seguinte corolário.

Corolário 1.2.1 Seja A o gerador infinitesimal de um $C^{\circ}$-semigrupo $T(t)$ satisfazendo

$$
\|T(t)\| \leq M e^{\omega t}
$$

Seja $\gamma>\max (0, \omega)$. Se $x \in D\left(A^{2}\right)$ então

$$
T(s) x d s=\frac{1}{2 \pi i} \int_{\gamma-i \infty}^{\gamma+i \infty} e^{\lambda t} R(\lambda: B) x d \lambda
$$

$e$, para $\delta \geq 0$, a integral imprópria converge uniformemente para $t \in\left[\delta, \frac{1}{\delta}\right]$.

Prova:

Seja $x \in D\left(A^{2}\right)$ então $A x \in D(A)$. Pelo Teorema anterior, temos que

$$
T(t) x-x=\int_{0}^{t} T(s) A x d s=\frac{1}{2 \pi i} \int_{\gamma-i \infty}^{\gamma+i \infty} e^{\lambda t} R(\lambda: A) A x \frac{d \lambda}{\lambda}
$$




$$
\begin{gathered}
=\frac{1}{2 \pi i} \int_{\gamma-i \infty}^{\gamma+i \infty} e^{\lambda t}\left(R(\lambda: A) x-\frac{x}{\lambda}\right) d \lambda \\
=\frac{1}{2 \pi i}\left(\int_{\gamma-i \infty}^{\gamma+i \infty} e^{\lambda t} \frac{d \lambda}{\lambda}\right) \cdot x+\frac{1}{2 \pi i} \int_{\gamma-i \infty}^{\gamma+i \infty} e^{\lambda t} R(\lambda: A)(x) d \lambda \\
=x+\frac{1}{2 \pi i} \int_{\gamma-i \infty}^{\gamma+i \infty} e^{\lambda t} R(\lambda: A)(x) d \lambda
\end{gathered}
$$

Pois $\frac{1}{2 \pi i}\left(\lim _{t \rightarrow \infty} \int_{\gamma-i t}^{\gamma+i t} e^{\lambda t} \frac{d \lambda}{\lambda}\right) x \longrightarrow x$. Portanto, temos a tese.

Podemos ainda estabelecer o seguinte resultado.

Teorema 1.2.2 Seja $T(t)$ um $C^{0}$-semigrupo satisfazendo $\|T(t)\| \leq M e^{\omega t}$. Seja $\gamma>\max (0, \omega)$. Se $x \in D\left(A^{2}\right)$ então

$$
T(s) x d s=\frac{1}{2 \pi i} \int_{\gamma-i \infty}^{\gamma+i \infty} e^{\lambda t} R(\lambda: B) x d \lambda .
$$

Concluimos esta seção com uma importante condição suficiente mas não necessária para que um operador $A$ seja um gerador infinitesimal de um $C^{0}$ semigrupo. As condições deste teorema são mais fáceis de se verificar, que as do Teorema 1.1.5

Teorema 1.2.3 (Pazy [15]) Seja A um operador densamente definido em $X$ satisfazendo as seguintes condições:

i)Para algum $0 \leq \delta \leq \frac{\pi}{2}$

$$
\rho(A) \supset \sum_{\delta}=\left\{\lambda:|\arg | \leq \frac{\pi}{2}+\delta\right\} \cup\{0\} .
$$

ii)Existe uma constante $M$ talque $\|R(\lambda: A)\| \leq \frac{M}{|\lambda|}$, para $\lambda \in \sum_{\delta}, \quad \lambda \neq 0$. Então A é um gerador infinitesimal de um $C^{0}-$ semigrupo $T(t)$, satisfazendo $\|T(t)\| \leq C$ para algum $C$.

Além disso,

$$
T(t)=\frac{1}{2 \pi} \int_{\gamma} e^{\lambda t} R(\lambda: A) d \lambda
$$

onde $\gamma$ é uma curva diferenciável em $\sum_{\delta}$ variando de $\infty e^{-i t}$ a $\infty e^{i t}$ para $\frac{\pi}{2} \leq \theta \leq \delta+\frac{\pi}{2}$ (ver figura 1.1). 


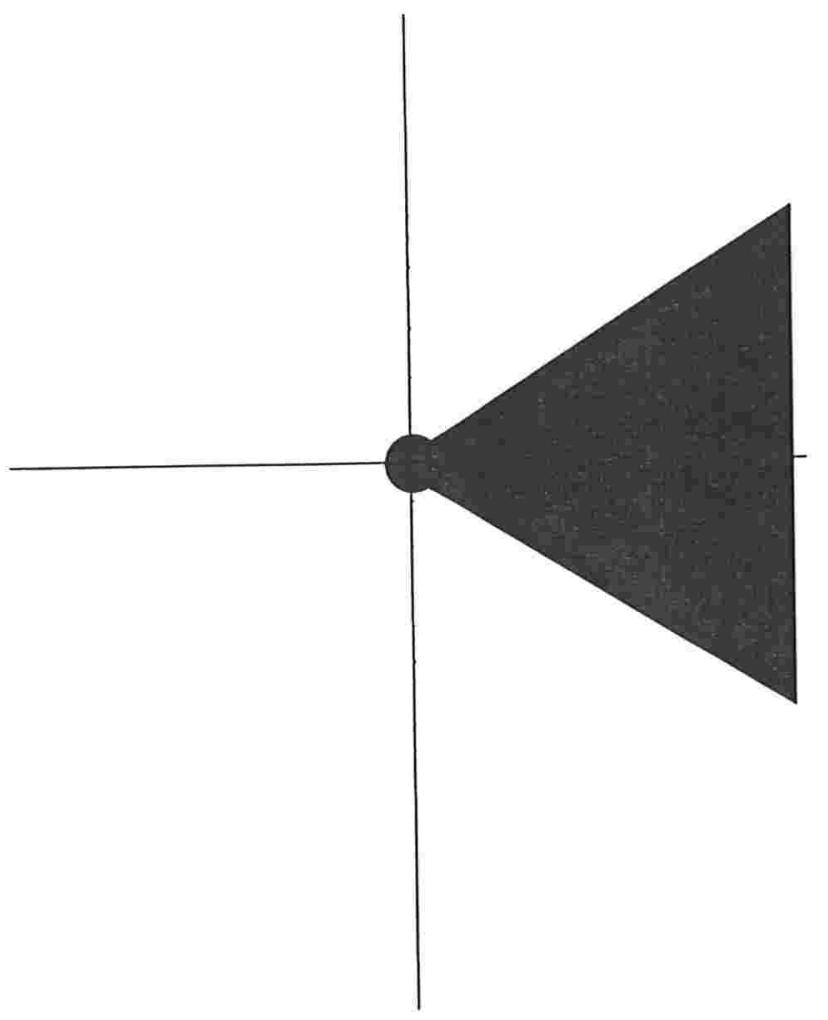

Figura 1.1:

\subsection{Operadores Setoriais, Potências Fracionárias e Semigrupos Analíticos.}

Nesta seção, apresentamos, a nosso ver, um dos teoremas mais importantes desta teoria. Nos referimos ao Teorema 1.3.1, cuja demostração pode ser encontrada em [11]. Este resultado é importante pois nos dá uma relação explícita entre o semigrupo (analítico) e seu gerador infinitesimal, além disso, ele é a solução de uma equação abstrata como veremos nos próximos capítulos.

Definição 1.3.1 Seja A um operador linear definido num espaço de Banach $X$. Dizemos que A é um operador setorial se é fechado, seu domínio é denso em $X$ e existem $\theta \in\left(0, \frac{2}{\pi}\right), M \geq 1$ e $a \in R$, de forma que o setor

$$
S_{b, \theta}=\{z \text { tal que } \theta \leq|\arg (z-b)| \leq \pi, \quad z \neq b\}
$$


está contido no conjunto resolvente de $A$ e

$$
\left\|(z I-A)^{-1}\right\| \leq \frac{M}{|z-b|}
$$

para todo $z \in S_{b, \theta}$.

Teorema 1.3.1 (D. Henry [11]) Se A é um operador setorial, então -A é o gerador infinitesimal do semigrupo analítico $\left\{e^{-A t}\right\}_{t \geq 0}$, onde

$$
e^{-A t}=\frac{1}{2 \pi} \int_{\gamma}(\lambda+A)^{-1} e^{\lambda t} d \lambda
$$

sendo $\gamma$ um contorno em $\rho(-A)$, com $\arg =\lambda \rightarrow \pm \theta$ quando $\lambda \rightarrow \infty$, para $\theta \in\left(\frac{\pi}{2}, \pi\right)$.

Além disso, $e^{- \text {At }}$ pode ser estendida analiticamente ao setor

$$
\{t \neq 0:|\arg t| \leq \epsilon\},
$$

contendo o eixo real positivo, e se $\operatorname{Re} \sigma(A)>a$ isto é, se $\operatorname{Re} \lambda \geq$ a para todo $\lambda \in \sigma(A)$, então para $t>0$ temos:

$$
\left\|e^{-A t}\right\| \leq C e^{-a t}, \quad\left\|A e^{-A t}\right\| \leq \frac{C}{t} e^{-a t} .
$$

Finalmente,

$$
\frac{d e^{-A t}}{d t}=-A e^{-A t} \quad t \geq 0
$$

Prova:

Faremos a prova de que $-A$ é um operador setorial, então, $A$ é o gerador infinitesimal de um semigrupo, dado pela forma resolvente:

$$
T(t)=\frac{1}{2 \pi i} \int_{\Gamma} e^{\lambda t} R(\lambda: A) d \lambda
$$

Sem perda de generalidade, assumimos que $a=0 \mathrm{e}$

$$
\left\|(\lambda-A)^{-1}\right\| \leq \frac{M}{|\lambda|+\delta}
$$


pois se $0 \in \rho(A)$ e se (1.5) é verdadeiro temos que $-A$ é setorial para $\theta \in\left(0, \frac{\pi}{2}\right)$, caso contrário, subtituimos $A$ por $A-a I$. Neste caso temos

$$
e^{-a t} T(t)=\frac{1}{2 \pi i} \int_{\Gamma_{a}} e^{(\lambda-a) t}(\lambda I-(A-a I))^{-1} d \lambda=\frac{1}{2 \pi i} \int_{\Gamma_{0}} e^{\mu t} R(\mu: A) d \mu
$$

onde $\mu=\lambda-a$.

Definamos $e^{t A}$ como a integral do enunciado.

Primeiro vejamos que a integral converge absolutamente, fazemos $\mu=$ $\lambda t$,

$$
\begin{aligned}
\left\|e^{A t}\right\| & \leq \frac{1}{2 \pi} \int_{\Gamma_{0}} e^{R e \mu t}\left\|\left(\frac{\mu}{t}-A\right)^{-1}\right\|\left|\frac{d \mu}{t}\right| \\
& \leq \frac{1}{2 \pi} \int_{\Gamma_{0}} e^{R e \mu t} \frac{M}{\frac{|\mu|}{t}}\left|\frac{d \mu}{t}\right| \\
& =2 \int_{\varepsilon}^{\infty} e^{r \cos \theta} \frac{M}{r} \theta d r \leq M .
\end{aligned}
$$

Pelo Teorema de Cauchy, a integral não muda se trasladamos a curva para a direita e trasformamos $\Gamma_{0}$ em $\Gamma_{1}$.

Então para $0 \leq t, 0 \leq s$

$$
\begin{aligned}
e^{A t} e^{s A} & =\frac{1}{(2 \pi i)^{2}} \int_{\Gamma_{0}} \int_{\Gamma_{1}} e^{\lambda t} e^{\mu t}(\lambda-A)^{-1}(\mu-A)^{-1} d \mu d \lambda \\
& =\frac{1}{(2 \pi i)^{2}} \int_{\Gamma_{0}} \int_{\Gamma_{1}} e^{\lambda t} e^{\mu t}(\mu-\lambda)^{-1}[R(\lambda: A)-R(\mu: A)] d \mu d \lambda
\end{aligned}
$$

$\operatorname{mas} \lambda \in \Gamma$ e $\mu \in \Gamma_{1}, \quad \int_{\Gamma_{0}} e^{\lambda t}(\mu-\lambda)^{-1} d \lambda=0$.

Fazemos o mesmo com $\int_{\Gamma_{1}} e^{\mu t}(\mu-\lambda)^{-1} d \mu=2 \pi i e^{\lambda s}$.

Então

$$
\begin{aligned}
e^{A t} e^{s A}= & \frac{1}{(2 \pi i)^{2}} \int_{\Gamma_{0}} \int_{\Gamma_{1}} e^{\lambda t} e^{\mu t}(\lambda-A)^{-1}(\mu-\lambda)^{-1} d \mu d \lambda \\
& +\frac{1}{(2 \pi i)^{2}} \int_{\Gamma_{0}} \int_{\Gamma_{1}} e^{\lambda t} e^{\mu t}(\mu-\lambda)^{-1}(\mu-A)^{-1} d \mu d \lambda \\
= & \frac{1}{(2 \pi i)^{2}} \int_{\Gamma_{0}} \int_{\Gamma_{1}} e^{\lambda t} e^{\mu t}(\lambda-A)^{-1}(\mu-\lambda)^{-1} d \mu d \lambda \\
= & e^{A(s+t)}
\end{aligned}
$$


Portanto, é um semigrupo.

Provemos agora que é um $\mathrm{C}^{0}$-semigrupo, fortemente contínuo, para isto é suficiente provar em um conjunto denso, que neste caso será $(D(A))$, pois $\left\|e^{A t}\right\| \leq C$, de fato, se $x \in D(A)$,

$$
\begin{aligned}
\left\|e^{t A} x-x\right\| & \leq \frac{1}{2 \pi} \int\left|e^{\lambda t}\right|\left|\lambda^{-1}\right|\left\|(\lambda-A)^{-1}\right\|\|A x\||d \lambda| \\
& \leq \frac{1}{2 \pi} \int_{\Gamma_{0}} e^{R e \mu}\left(\frac{|\mu|}{t}\right) \frac{\|A x\|}{\frac{|\mu|}{t}} \frac{d \mu}{t} \\
& \leq \frac{t}{2 \pi} \int_{\Gamma_{0}} e^{R e \mu} \frac{\|A x\|}{|\mu|^{2}} d \mu=o(t) .
\end{aligned}
$$

Portanto, $\mathrm{T}(\mathrm{t})$ é um semigrupo fortemente contínuo, o qual pode ser estendido a um semigrupo analítico o conjunto $\{t \neq 0, \quad|\arg t|<\epsilon\}$ (ver [15]). Provemos que $\frac{d e^{t A}}{d t}=A e^{A t}$.

$$
\begin{aligned}
\frac{d e^{t A}}{d t}-A e^{A t} & =\frac{1}{2 \pi i} \int_{\Gamma} e^{\lambda t}(\lambda-A)^{-1} d \lambda-\frac{1}{2 \pi i} \int_{\Gamma} e^{\lambda t} A(\lambda-A)^{-1} d \lambda \\
& =\frac{1}{2 \pi i} \int_{\Gamma} e^{\lambda t}(\lambda-A)(\lambda-A)^{-1} d \lambda=0
\end{aligned}
$$

Se $x \in D(A)$, sabemos que

$$
T(t) x-T(s) x=\int_{s}^{t} T(u) A x d u
$$

e

$$
\lim _{h \rightarrow 0} \frac{1}{h} \int_{t}^{t+h} T(u) d u=T(t) x
$$

Portanto,

$$
\frac{e^{t A} x-x}{t}=\frac{1}{t} \int_{0}^{t} T(u) A x d u \longrightarrow A x
$$

logo, o gerador $A$ está contido no gerador $G$ de $T(A x=G x \forall x \in D(A))$. Agora definamos para $\lambda \geq 0$

$$
R(\lambda) x=\int_{0}^{\infty} e^{-\lambda t} e^{-A \lambda t} d t
$$

Para qualquer $x, e^{-A \lambda t} x \in D(A)$, para $t>0$ temos

$$
A \int_{\delta}^{\infty} e^{-\lambda t} e^{-A \lambda t} d t=e^{-\lambda \delta} e^{-A \delta}-\lambda \int_{\delta}^{\infty} e^{-\lambda t} e^{-A t} d t
$$


Como $A$ é fechado segue que $R(\lambda) x \in D(A) \in D(G) \forall \lambda \geq 0, x \in X$. De maneira similar se mostra que

$$
R(\lambda)(\lambda-G) x=x \text { para } x \in D(G) .
$$

Então $D(G) \subset$ posto de $R(\lambda) \subset D(A)$, o que mostra que $-A=G$.

Para o estudo das equações diferenciais parciais semilineares, seram introduzidos alguns espaços relacionados com o operador $A$ e suas "potências". Sua importância ficará claro logo.

\subsection{Expoente Fracionário de um Operador.}

Seja $A$ um operador fechado densamente definido em espaço de Banach $X$, tal que seu conjunto resolvente inclui $(-\infty, 0]$ e $\left\|(\lambda I-A)^{-1}\right\| \leq M, \lambda \leq 0$. Então definimos para $\alpha \geq 0$

$$
A^{-\alpha}=\frac{1}{2 \pi} \int_{\gamma} s^{-\alpha}(\lambda-A)^{-1} d \lambda
$$

onde $\lambda^{-\alpha}:=|\lambda|^{\alpha} e^{-i \alpha \arg \lambda} \quad$ e $\gamma$ é um contorno em $\rho(A)$ em torno do eixo real negativo.

Além disso, assumiremos a seguinte propriedade:

$$
\rho(A) \supset \sum_{\delta}=\{\lambda: \omega \leq|\arg \lambda| \leq \pi\} \cup V,
$$

onde $V$ é uma vizinhança de 0 .

Se $\mathrm{M}=1$ e $\omega=\frac{\pi}{2}$ então $-A$ é o gerador infinitesimal de um $\mathrm{C}^{0}$-semigrupo. Se $\omega<\frac{\pi}{2}$ então, pelo Teorema 1.2.3, -A é o gerador infinitesimal de um semigrupo analítico.

A integral converge na topologia uniforme de operadores, para todo $\alpha \geq$ 0 . Portanto, define um operador linear limitado $A^{-\alpha}$. Se $\alpha=+n$ natural, o integrando é analítico em $\sum_{\delta}$ e é fácil ver que a curva de integração pode ser transformada em um círculo pequeno em torno da origem. Então, usando o teorema dos resíduos, segue que a integral é igual a $A^{-n}$.

Se $\omega<\frac{\pi}{2}$, isto é, se -A é o gerador infinitesimal de um semigrupo analítico $T(t)$, podemos obter uma representação de $A^{-\alpha}$ que é muito útil e é a que utilizaremos. Como supomos que $0 \in \rho(A)$, existe uma constante $\delta \geq 0$, tal 
que $-A+\delta$ é o gerador infinitesimal de um semigrupo analítico. Isto implica que:

$$
\begin{aligned}
& \|T(t)\| \leq M e^{-\delta t}, \\
& \|A T(t)\| \leq M_{1} t^{-1} e^{-\delta t}, \\
& \left\|A^{m} T(t)\right\| \leq M_{m} t^{-m} e^{-\delta t} .
\end{aligned}
$$

Além disso, sabemos que $(t I+A)^{-1}=\int_{0}^{\infty} e^{-s t} T(s) d s$ converge uniformemente para $t \geq 0$, na topologia uniforme dos operadores pois

$$
\|T(t)\| \leq M e^{-\delta t}
$$

logo, temos

$$
A^{-\alpha}=\frac{1}{\Gamma(\alpha)} \int_{0}^{\infty} T(s) s^{\alpha-1} d s
$$

onde a integral converge na topologia uniforme dos operadores, para todo $\alpha \geq 0$.

Os seguintes lemas fornecem algumas propriedades que justificam a definição de $A^{\alpha}$ como potência de $A$.

Lema 1.4.1 Para $\alpha>0$, e $\beta>0$, temos $A^{-(\alpha+\beta)}=A^{-\alpha} A^{-\beta}$.

Lema 1.4.2 Existe uma constante $C$ tal que $\left\|A^{-\alpha}\right\| \leq C$, para $0<\alpha<1$.

Lema 1.4.3 Para todo $x \in X$, temos $\lim _{\alpha \rightarrow 0} A^{-\alpha} x=x$.

Corolário 1.4.1 Se A satisfaz (1.6) com $\omega<\frac{\pi}{2}$ então $A^{-1}$ é um $C^{0}$ semigrupo de operadores lineares limitados.

Lema 1.4.4 $A^{-\alpha}$ é injetivo.

Definição 1.4.1 Seja A com as hipóteses anteriores e $\omega<\frac{\pi}{2}$. Para todo $\alpha>0$, definimos

$$
A^{\alpha}=\left(A^{-\alpha}\right)^{-1} \text {. }
$$

Teorema 1.4.1 (Pazy [15]) Seja $A^{\alpha}$ definido como antes. Então

i) $A^{\alpha}$ é um operador fechado com domínio $D\left(A^{\alpha}\right)=R\left(A^{-\alpha}\right)$,

ii) $\alpha \geq \beta>0$ então $D\left(A^{\alpha}\right) \subset D\left(A^{\beta}\right)$,

iii) $\overline{D\left(A^{\alpha}\right)}=X, \quad$ para todo $\quad \alpha \geq 0$. 


\subsection{Exemplos}

(i) Se $A$ é um escalar positivo, então $A^{-\alpha}$ é definida na forma usual como a $(-\alpha)$ potência de $A$.

(ii) Se $A$ é definido positivo, autodjunto em um espaço de Hilbert com representação espectral $A=\int_{0}^{\infty} \lambda d E$, então $A^{-\alpha}=\int_{0}^{\infty} \lambda^{-\alpha} d E$.

(iii) $A^{-1}$ é o inverso de $A$. 


\section{Capítulo 2}

\section{O Problema de Cauchy}

Queremos estudar o problema de Cauchy em espaços de Banach, a teoria aqui apresentada pode ser encontrada em [11]. Primeiro, consideremos o problema homogêneo:

$$
\frac{d x}{d t}+A x=0 \quad x(0)=x_{0}
$$

onde $A$ é um operador setorial num espaço de Banach $X, x_{0} \in X$ dado, $x:[0, t] \rightarrow X$.

Definição 2.0.1 Uma solução do problema (2.1) é uma função contínua $x:[0, t] \rightarrow X$, continuamente diferenciável em um intervalo aberto $(0, t)$, $x(t) \in D(A)$, para $0<t<T$, satisfaz a equação em $(0, t)$ e $x(t) \rightarrow x_{0}$, quando $t \rightarrow 0^{+}$.

Do teorema (1.3.1), como $A$ é setorial, é claro que $x(t)=e^{-t A} x_{0}$ é uma solução da equação (2.1), falta provar que é a única.

Seja $0 \leq s \leq t<T$ e $y(t, s)=e^{-A(t-s)} x(s)$, onde $x($.$) é uma solução$ qualquer da equação em $[0, t]$, então a aplicação $s \rightarrow y(t, s)$ é contínua em $0 \leq s \leq t$ e continuamente diferenciável em $0<s \leq t \quad$ com

$$
\frac{\partial y}{\partial s}=e^{-A(t-s)} \frac{d x}{d s}+A e^{-A(t-s)} x(s)=0
$$

então, para $0<s<t, \quad y(t, t)=y(t, 0)=e^{-A t} x(0)$.

Agora consideramos o problema não homogêneo:

$$
\frac{d x}{d t}+A x=f(t) \quad 0<t<T \quad x(0)=x_{0}
$$


Lema 2.0.1 Seja $f:(0, t) \rightarrow X$, uma função localmente Hölder contínua, com $\int_{0}^{\rho}\|f(s)\| d s<\infty$, para algum $\rho>0$. Para $0<t<T$ consideremos

$$
F(t)=\int_{0}^{t} e^{-A(t-s)} f(s) d s .
$$

Então $F(t)$ é contínua em $[0, T)$, continuamente diferenciável em $(0, T)$ com $F(t) \in D(A)$, para $0<t<T$, e $\frac{d F}{d t}+A F=F(t), 0<t<T$ e $F(t) \rightarrow 0 \mathrm{em}$ $X$ quando $t \rightarrow 0^{+}$.

Teorema 2.0.1 Suponhamos A um operador setorial em $X, x_{0} \in X, f$ : $(0, t) \rightarrow X$ localmente Hölder contínua com $\int_{0}^{\rho}\|f(s)\| d s<\infty, \quad$ para algum $\rho>0$, então existe uma única solução $x($.$) de$

$$
\begin{aligned}
\frac{d x}{d t}+A x & =f(t) \quad 0<t<T \\
x(0) & =x_{0}
\end{aligned}
$$

dada pela seguinte fórmula

$$
x(t)=e^{-A t} x_{0}+\int_{0}^{t} e^{-A(t-s)} f(s) d s .
$$

A prova segue do Lema anterior e da solução de problema homogêneo.

\subsection{Existência local e unicidade}

Agora consideremos a equação semilinear

$$
\begin{aligned}
& \frac{d x}{d t}+A x=f(t, x), \quad t_{0}<t \\
& x(0)=x_{0}
\end{aligned}
$$

onde assumimos que $A$ é um operador setorial tal que as potências fracionárias de $A_{1}=A+a I$ estão bem definidas para algum $a \in R$ e os espaços

$$
X^{\alpha}=D\left(A_{1}^{\alpha}\right),
$$

com a norma do gráfico

$$
\|x\|_{X^{\alpha}}=\left\|A_{1}^{\alpha} x\right\|
$$

estão definidos para $\alpha \geq 0$. Seja $U \subset R \times X^{\alpha}, 0 \leq \alpha<1$ e $f: U \rightarrow X$ uma função localmente Hölder contínua em $t$ e localmente Lischitziana em $x$, ou 
seja, para cada $\left(t_{0}, x_{0}\right) \in U$, existe uma vizinhança do $V \subset U$ de $\left(t_{0}, x_{0}\right)$, tal que para todo $(s, t) \in V$ temos

$$
\|f(t, x)-f(t, y)\| \leq M\left(|t-s|^{\beta}+\|x-y\|_{\alpha}\right) .
$$

Definição 2.1.1 Uma solução da equação não linear em $\left[t_{0}, \tau\right)$ é uma função contínua $x:\left[t_{0}, \tau\right) \rightarrow X^{\alpha}$, com $x\left(t_{0}\right)=x_{0}$, tal que $f(t, x()):$. $\left[t_{0}, \tau\right) \rightarrow X^{\alpha}$ é continua, $x(t) \in D(A), \frac{d x(t)}{d t}$, existe, $t \rightarrow f(t, x(t))$ é localmente Hölder contínua,

$$
\int_{0}^{t}(t-s)^{-\alpha+\epsilon}\|f(s, x(s))\| d s \rightarrow 0
$$

e satisfaz (2.3) em $\left[t_{0}, \tau\right)$.

O problema de Cauchy consiste em dado um $x_{0} \in X^{\alpha}$ encontrar uma solução para (2.3).

Lema 2.1.1 Se x é solução de (2.3) em $\left[t_{0}, t_{1}\right)$, então

$$
x(t)=e^{-A\left(t-t_{0}\right)} x_{0}+\int_{0}^{t} e^{-A(t-s)} f(s, x(s)) d s .
$$

Reciprocamente, se $x$ é uma função contínua de $\left(t_{0}, t_{1}\right)$ em $X^{\alpha}$,

$$
\int_{0}^{t}(t-s)^{-\alpha+\epsilon}\|f(s, x(s))\| d s \rightarrow 0
$$

e, além disso, a equação integral é satisfeita para $t \in\left(t_{0}, t_{1}\right)$, então $x(t)$ é a solução da equação diferencial (2.3) em $\left(t_{0}, t_{1}\right)$.

Teorema 2.1.1 Suponhamos que $A$ é um operador setorial, $x_{0} \in X, f$ : $U \rightarrow X$, onde $U$ é um aberto de $R \times X^{\alpha}$, localmente Hölder contínua em $t$ e localmente Lipschitz em $x$, então para $\left(t_{0}, x_{0}\right) \in U$ existe $T=T\left(t_{0}, x_{0}\right)$ tal que (2.4) possue uma única solução com valor incial $x(0)=x_{0}$.

Como em equações diferenciais ordinárias, podemos encarar a solução do problema de Cauchy como a solução de uma equação integral, dita normalmente "fórmula da variações das constantes" para a equação (2.3).

Por último necessitaremos (nas aplicaçōes) de um teorema que garanta a existência da solução para todo tempo, o teorema é o seguinte 
Teorema 2.1.2 (D. Henry [11]) Suponhamos A um operador setorial, $f: U \rightarrow X U=(\tau, \infty) \times X^{\alpha}$ localmente Hölder contínua em $t$, localmente Lipschitz em $x$ para $(t, x) \in U$ e

$$
\|f(t, x)\| \leq K(t)\left(1+\|x\|_{\alpha}\right)
$$

$\forall(t, x) \in U$ onde $K($.$) é contínua em (\tau, \infty) \times X^{\alpha}$. Se $\tau_{0}>\tau, x_{0} \in X^{\alpha}$ existe uma única solucão de (2.3) por $\left(x_{0}, t_{0}\right)$ para todo $t \geq T_{0}$.

\subsection{Estabilidade e instabilidade dada pela primei- ra aproximação}

Com os resultados de existência e unicidade, agora nos concentraremos nos resultados de estabilidade. Aqui enunciamos dois resultados referentes à estabilidade das solucões do problema de Cauchy que serão utilizados no capítulo 5. Estes resultados podem ser encontrados em [11].

Sejam $\tau \in R, x_{0} \in X^{\alpha}$ e $A$ um operador setorial em um espaço de Banach $X$ e $f: U \rightarrow X$, onde $U$ é uma vizinhança cilíndrica em $R \times X^{\alpha}$, $\alpha<1$, de $(\tau, \infty) \times\left\{x_{0}\right\}$.

Definição 2.2.1 Dizemos que um ponto $x_{0} \in X^{\alpha}$ é um ponto de equilibrio, se $x(t)=x_{0}$ é uma solução de $\frac{d x}{d t}+A x=f(t, x), t_{0}<t$.

Definição 2.2.2 Uma solução $\bar{x}($.$) em \left[t_{0}, \infty\right)$ é estável em $X^{\alpha}$ se para todo $\epsilon>0$, existe $\delta>0$ tal que para qualquer solução $x$ com $\left\|x\left(t_{0}\right)-\bar{x}\left(t_{0}\right)\right\|_{\alpha}<\delta$ existe em $\left[t_{0}, \infty\right)$ e satisfaz $\|x(t)-\bar{x}(t)\|_{\alpha}<\epsilon, t \geq t_{0}$.

Definição 2.2.3 Uma solução $\bar{x}($.$) é uniformemente estável se x_{1} \rightarrow x\left(t, t_{1}, x_{1}\right)$ é contínua quando $x_{1} \rightarrow \bar{x}\left(t_{1}\right)$, uniformemente em $t \geq t_{0}$ e $t_{1} \geq t_{0}$.

Definição 2.2.4 Uma solução $\bar{x}($.$) é uniformemente assintóticamente estável$ se é uniformemente estável e $x\left(t, t_{1}, x_{1}\right)-\bar{x}(t) \rightarrow 0$ quando $t-t_{1} \rightarrow \infty$ uniformemente $\epsilon m t_{1} \geq t_{0} e\left\|x_{1}-\bar{x}\left(t_{1}\right)\right\|_{\alpha}<\delta$, para alguma constante $\delta>0$.

Teorema 2.2.1 (D. Henry [11]) Sejam A, $f$, como antes, e seja $x_{0} u m$ ponto de equilibrio. Suponhamos que

$$
f\left(t, x_{0}+z\right)=f\left(t, x_{0}\right)+B z+g(t, z)
$$

onde $B$ é um operador linear limitado de $X^{\alpha}$ em $X$, e $\|g(t, z)\|=o\left(\|z\|_{\alpha}\right)$ quando $\|z\|_{\alpha} \rightarrow 0$, uniformemente em $t>t_{0}$, e $f(t, z)$ é localmente Hölder 
em $t$ e localmente Lipschitziana em $x$ em $U$. Se o espectro de $A-B$ se encontra em $\{$ Re $\lambda>\beta\}$, ou equivalentemente, a linearização $\frac{d x}{d t}+A x=B x$ é uniformemente assintóticamente estável, então a equação original tem $x_{0}$ como uma solução unifomemente asintóticamente estável em $X^{\alpha}$.

Teorema 2.2.2 (D. Henry [11]) Seja A um operador setorial onde $f(t, x)$ é localmente Lipschitz em $x$, Hölder contínua em $t$ e $f$ leva um entorno cilíndrico de $R \times\left\{x_{0}\right\}$ em $R \times X^{\alpha}$. Suponha que

$$
A x_{0}=f\left(t, x_{0}\right),
$$

para $t \geq t_{0}$,

$$
\begin{aligned}
f\left(t, x_{0}+z\right) & =f\left(t, x_{0}\right)+B z+g(t, z), g(t, 0)=0 \\
\left\|g\left(t, z_{1}\right)-g\left(t, z_{2}\right)\right\| & \leq k(\rho)\left\|z_{1}-z_{2}\right\|_{\alpha}, \text { para }\left\|z_{1}\right\| \leq \rho,\left\|z_{2}\right\| \leq \rho,
\end{aligned}
$$

e $k(\rho) \rightarrow 0$ quando $\rho \rightarrow 0^{+}$. Seja $L=A-B$, e suponhamos que $\sigma(L) \cap$ $\{R e \lambda<0\}$ é um conjunto espectral não vazio, então o ponto de equilíbrio $x_{0}$ e instável.

É bem conhecida a importância do teorema da primeira aproximação para equações diferenciais ordinárias, com o teorema anterior temos o mesmo resultado para equações diferenciais parciais.

\subsection{Exemplo}

Consideremos

$$
\begin{gathered}
\frac{\partial u}{\partial t}-\frac{\partial u}{\partial x}=\frac{\partial^{2} u}{\partial x^{2}}+f(t, x, u) \\
u(0, t)=0, u(\pi, t)=0 \text { em } \pi>x>0, t>0
\end{gathered}
$$

onde $f: R^{+} \times[0, \pi] \rightarrow 0$ é mensurável em $x$, localmente Hölder contínua em $t$ e localmente Lipschitz em $u$, uniformemente em $x \operatorname{com}|f(t, x, u)| \leq$ $h(x) g(t,|u|), h \in L^{2}(0, \pi), g$ contínua e crescente.

Tomemos $X=L^{2}(0, \pi), A=-\frac{d^{2}}{d x^{2}}$, com domínio em $H^{2}(0, \pi) \cap H_{0}^{1}(0, \pi)$, e

$$
D\left(A^{\frac{1}{2}}\right)=X^{\frac{1}{2}}=H_{0}^{1}(0, \pi)
$$

pode-se provar (ver ([11]) que $F: R^{+} \times H_{0}^{1}(0, \pi) \rightarrow L_{0}^{2}(0, \pi)$, dada por

$$
F(t, v(x))=-v^{\prime}(x)+f(t, x, v(x))
$$

satisfaz as hipóteses do teorema 2.1.2. 


\section{Capítulo 3}

\section{Atratores e Conjuntos Invariantes para $C^{r}$-semigrupos}

Neste capítulo estão reunidos alguns resultados encontrados em [9]. Eles generalizam os resultados em dimensão finita, por exemplo de $\omega$-limite, $\alpha$ limite, atrator global, órbita, e serão usados nos capítulos 4 e 5 .

Definição 3.0.1 Para $x \in X$ e $T(t): X \rightarrow X, t \geq 0$, um $C^{r}$ - semigrupo $r \geq 0$, definimos a órbita positiva $\gamma^{+}(x)$ por $x$, como : $\gamma^{+}(x)=\{T(t) x$, $t \geq 0\}$.

Uma órbita negativa por $x$ é uma função $\phi:[0, \infty) \rightarrow X$ tal que $\phi(0)=x$ e $\forall \mathrm{s} \leq 0, \mathrm{~T}(\mathrm{t}) \phi(s)=\phi(t+s)$ para $0 \leq t \leq-s$. Denotaremos por $\gamma^{-}(x)$ a reunião de todas as órbitas negativas por $x$, ou seja,

$$
\gamma^{-}(x)=\bigcup_{t \geq 0} H(t, x)
$$

onde $H(t, x)=\{y \in X \quad / \exists$ uma órbita negativa por $x, \phi$, com $\phi(0)=x$ e $\phi(-t)=y\}$. Tal conjunto também será denotado por $\gamma^{-}(x)$ como a órbita negativa por $x$. Dizemos que existe uma órbita completa por $x$ através de $T(t), t \geq 0$ quando existe uma função $\phi: R \rightarrow X$ tal que $\phi(0)=x \mathrm{e}$, para qualquer $\mathrm{s} \in R$, temos $T(t) \phi(s)=\phi(t+s)$, para $t \geq 0$. Definimos $\gamma(x)=\gamma^{-}(x) \cup \gamma^{+}(x)$.

Para $B \subset X$, definimos $\gamma^{+}(B)=\bigcup_{x \in B} \gamma^{+}(x), H(t, B)=\bigcup_{x \in B} H(t, x)$, 
$\gamma^{-}(B)=\bigcup_{x \in B} \gamma^{-}(x), \gamma(B)=\bigcup_{x \in B} \gamma(x)$

Definição 3.0.2 Para um conjunto $B \subset X$ definimos o conjunto $w$-limite de $B$ e conjunto $\alpha$-limite de $B$, denotados por $w(B)$ e $\alpha(B)$, respectivamente, da seguinte maneira:

$$
w(B)=\bigcap_{s \geq 0} \overline{\bigcup_{t \geq s} T(t) B}, \quad \alpha(B)=\bigcap_{s \geq 0} \overline{\bigcup_{t \geq s} H(t, B)}
$$

Definição 3.0.3 Uma $\varepsilon$-vizinhança de um subconjunto $B \subset X$ é o conjunto aberto $B=$ definido por

$$
B_{\varepsilon}=\bigcup_{x \in B} B_{\varepsilon}, \text { onde } B_{\varepsilon}=\{y \in X /\|y-x\|<\varepsilon\},
$$

Dizemos que um conjunto $\mathrm{B} \subset X$ atrai um conjunto $\mathrm{C} \subset X$ através de $\mathrm{T}(\mathrm{t})$ se $\operatorname{dist}(\mathrm{T}(\mathrm{t}) \mathrm{C}, \mathrm{B}) \rightarrow 0$, quando $\mathrm{t} \rightarrow \infty$, onde

$$
\operatorname{dist}(A, B)=\sup _{y \in A} \inf _{x \in B} d(x ; y) .
$$

Definição 3.0.4 Um conjunto $S \subset X$ é dito invariante se $\forall x \in S$ existe uma órbita completa por $x$ contida em $S$.

Definição 3.0.5 Sejam $T(t): X \rightarrow X, t \geq 0$, um $C^{0}$-semigrupo e $\mathcal{A} \subset \mathcal{X}$, A um subconjunto compacto e invariante através de $T(t)$. Dizemos que $\mathcal{A}$ é compacto invariante maximal para $T(t)$ se todo subconjunto $B \subset X$ compacto e invariante por $T(t), t \geq 0$, está contido em $\mathcal{A}$.

Definição 3.0.6 Um conjunto compacto invariante maximal para um $C^{r}$ semigrupo em $X, T(t), t \geq 0, r \geq 0$ é chamado atrator global para $T(t)$, $t \geq 0$ se atrai subconjuntos limitados de $X$.

Observação 3.0.1 Segue diretamente da definição que se existe um conjunto de $X$ compacto invariante maximal para $T(t), t \geq 0$, então é único.

Definição 3.0.7 $T(t): X \rightarrow X, t \geq 0$ é assintoticamente liso se para todo conjunto não vazio, limitado $B \subset X$ para o qual $T(t) B \subset B$, existe um conjunto $J \subset B$ compacto tal que $J$ atrai $B$.

Definição 3.0.8 Um semigrupo se chama disipativo ponto a ponto se existe um conjunto limitado $B \subset X$ tal que atrai pontos de $X$ através de $T(t)$. 
Teorema 3.0.1 (J. Hale [9]) Se $T(t): X \rightarrow X, t \geq 0$ é assintoticamente liso, dissipativo ponto a ponto e as órbitas dos conjuntos limitados são limitadas, então, existe um atrator global $\mathcal{A}$. Se $T(t)$ é injetor em $\mathcal{A}$ então $\left.T(t)\right|_{\mathcal{A}}$ é um $C^{r}-$ semigrupo.

A importância dos resultados anteriores é que eles nos permitem obter toda a informação dinâmica da equação olhando somente para o atrator global, isto é, passado algum tempo, a solução se comporta como a solução restrita ao atrator. 


\section{Capítulo 4}

\section{Atratores para Problemas Parabólicos com Condição de Fronteira não Linear}

\subsection{Introdução}

Seja $\Omega$ um domínio diferenciável do $R^{n}$. Neste capítulo consideramos o sistema de reação difusão, com disperção, dado pelo sistema

$$
\left\{\begin{array}{c}
u_{t}=\operatorname{Div}(a \nabla u)+\sum_{j=1}^{n} B_{j}(x) \frac{\partial u}{\partial x_{j}}+\lambda u-f(u) \quad \text { em } \Omega \\
\frac{\partial v_{i}}{\partial n_{\alpha}}=g(u) \quad \text { em } \partial \Omega
\end{array}\right.
$$

onde $u=\left(u_{1}, \ldots, u_{N}\right), N \leq 1, \lambda>0, a(x)=\operatorname{diag}\left(a_{1}(x), \ldots, a_{N}(x)\right), a_{i} \in$ $C^{1}(\Omega)$,

$0 \leq a_{i}(x)$. Sejam $f=\left(f_{1}, \ldots \ldots . ., f_{N}\right) \in C^{1}\left(R^{N}, R^{N}\right)$ e $g=\left(g_{1}, \ldots \ldots . ., g_{N}\right) \in$ $C^{2}\left(R^{N}, R^{N}\right)$. Se $f$ é a fonte do calor e $g=0$ temos que a solução explode em tempo infinito. O mais importante deste trabalho é o controle do crescimento do calor por meio do fluxo dissipativo pela fronteira.

O que pretendemos fazer é definir uma equação abstrata cuja solução "fornece" soluções da equação (4.1), como é feito no estudo de equações diferenciais parciais com condição de fronteira Neumann, para isto precisamos de alguns espaços e operadores especiais, seguiremos aqui os resultados encontrados em [18]. 


\subsection{Espaços de Lebesgue}

Seja $S=S\left(R^{n}\right)$ o espaço de todas as funções complexas rapidamente decrescentes infinitamente diferenciávies no espaço n-dimensional real $R^{n}$. Como é usual, $S^{\prime}=S^{\prime}\left(R^{n}\right)$ denota o espaço das distribuições temperadas que é o dual de $S$. Seja

$$
F(\Phi)(\varepsilon)=\frac{1}{(2 \pi)^{\frac{n}{2}}} \int_{R^{n}} e^{-i\langle x, s\rangle} \Phi(x) d x, \Phi \in S
$$

a transformada de Fourier de $\Phi$ e

$$
F^{-1}(\Phi)(\varepsilon)=\frac{1}{(2 \pi)^{\frac{n}{2}}} \int_{R^{n}} e^{i\langle x, \varepsilon\rangle} \Phi(x) d x,
$$

a tranformada inversa de $\Phi$.

Com isso podemos definir os espaços de Lebesgue em $R^{n}$, como segue:

Definição 4.2.1 Sejam $-\infty<s<\infty$ e $1<p<\infty$. Então

$$
H_{p}^{s}\left(R^{n}\right)=\left\{f: f \in S^{\prime}\left(R^{n}\right),\|f\|_{H_{p}^{s}\left(R^{n}\right)}=\left\|F^{-1}\left(1+|x|^{\frac{3}{2}} F f\right)\right\|_{L^{p}}<\infty\right\} .
$$

O primeiro resultado relaciona os expoentes negativos com os espaços duais

Teorema 4.2.1 Sejam $-\infty<s<\infty$ e $1<p<\infty$. Então

$$
\left(H_{p}^{s}\left(R^{n}\right)\right)^{\prime}=H_{q}^{-s}\left(R^{n}\right), \quad \frac{1}{p}+\frac{1}{q}=1 .
$$

O resultado seguinte mostra a relação entre os espaços de Sobolev $\left(W^{1, p}\right)$ e os espaços de Lebesgue.

Teorema 4.2.2 Sejam $-\infty<s_{0}, s_{1}<\infty \quad$ e $1<p_{0}, p_{1}<\infty$. Então $W^{s_{0}, p_{0}}\left(R^{n}\right)=H_{p_{1}}^{s_{1}}\left(R^{n}\right)$ se e somente se $s_{0}=s_{1}$ é um inteiro e $p_{0}=p_{1}$, ou $s_{0}=s_{1}$ e $\quad p_{0}=p_{1}=2$.

Agora definimos os espaços de Lebesgue com domínio $\Omega \subset R^{n}$.

Definição 4.2.2 Seja $\Omega \subset R^{n}$ um domínio arbitrário (limitado ou não), além disso sejam $-\infty<s<\infty$ e $1<p<\infty$. Então $H_{p}^{s}(\Omega)$ é a restrição de $H_{p}^{s}\left(R^{n}\right)$ a $\Omega$ com a norma 


$$
\|f\|_{H_{p}^{s}(\Omega)}=\inf _{\left.g\right|_{\Omega}=f}\|g\|_{H_{p}^{s}\left(R^{n}\right)}, \quad g \in H_{p}^{s}\left(R^{n}\right) .
$$

Observação 4.2.1 $H_{p}^{s}\left(R^{n}\right)$ é um espaço de Banach, para todo s.

Existem outras formas de restringir $H_{p}^{s}\left(R^{n}\right)$ a $\Omega$.

Definição 4.2.3 Seja $\Omega \subset R^{n}$ um domínio $C^{\infty}$. Além disso, sejam $-\infty<s<\infty$ e $1<p<\infty$. Então

$$
\begin{aligned}
\widehat{H_{p}^{s}(\Omega)} & =\left\{f: f \in H_{p}^{s}\left(R^{n}\right), \text { supp } f \subset \bar{\Omega}\right\} \\
\widehat{H_{p}^{s}\left(R^{n}\right)} & =\text { completamento de } C_{0}^{\infty}(\Omega) \text { em } H_{p}^{s}\left(R^{n}\right)
\end{aligned}
$$

O seguinte teorema estabelece as relações entre os espaços anteriores.

Teorema 4.2.3 Seja $\Omega \subset R$ um domínio $C^{\infty}$. Então

i) $\operatorname{Se} 1<p<\infty \quad$ e $-\infty<s<\frac{1}{p} \quad$ então $H_{p}^{s}(\Omega)=\widehat{H_{p}^{s}(\Omega)}$ ii) Se $-\infty<s<\infty, 1<p<\infty$ e $1 \leq q<\infty$ então $C_{0}^{\infty}(\Omega)$ é denso em $\widehat{H_{p}^{s}(\Omega)}$ e se verifica que $\widehat{H_{p}^{s}(\Omega)} \subset \widehat{H_{q}^{s}(\Omega)}$.

iii) Se $1<p<\infty$ e $\frac{1}{p}-1<s<\infty$ com $s-\frac{1}{p} \neq$ inteiro, então $\widehat{H_{p}^{s}(\Omega)}=\widehat{H_{p}^{s}(\Omega)}$.

As relações de dualidade são dadas pelo teorema seguinte.

Teorema 4.2.4 Seja $\Omega \subset R^{n}$ um domínio arbitrário, $-\infty<s<\infty$, e $1<p<\infty$, então

$$
\left.\widetilde{\left(H_{p}^{s}(\Omega)\right.}\right)^{\prime}=H_{q}^{-s}(\Omega)
$$

Teorema 4.2.5 Seja $\Omega \subset R^{n}$ um domínio $C^{\infty}$..

i) Se $1<p<\infty$ e $\frac{1}{p}-1<s<\infty$ com $s-\frac{1}{p} \neq$ inteiro então $\widehat{\left(H_{p}^{s}(\Omega)\right)^{\prime}}=H_{q}^{-s}(\Omega)$.

ii) Se $1<p<\infty$ e $\quad \frac{1}{p}-1<s<\frac{1}{p}$ então $\left(H_{p}^{s}(\Omega)\right)^{\prime}=H_{q}^{-s}(\Omega)$.

iii) $\operatorname{Se} 1<p<\infty \quad e \quad-\infty<s<\frac{1}{p} \operatorname{então}\left(H_{p}^{s}(\Omega)\right)^{\prime}=\widetilde{H_{q}^{-s}(\Omega)}$.

Também necessitamos de alguns resultados sobre a relação destes espaços com outros espaços conhecidos. 
Definição 4.2.4 i) Seja $t \geq 0$ um inteiro, então $C^{t}\left(R^{n}\right)$ é o completamento de $S\left(R^{n}\right)$ com a norma

$$
\|f\|_{C^{t}}=\sum_{|\alpha| \leq t} \sup _{x \in R^{n}}\left\|D^{\alpha} f(x)\right\| .
$$

ii) Se $0<[t]+\{t\}$ onde $[t]$ é a parte inteira de $t$, então

$$
C^{t}\left(R^{n}\right)=\left\{f: f \in C^{t}\left(R^{n}\right),\|f\|_{C^{t}}<\infty\right\},
$$

onde

$$
\|f\|_{C^{t}}=\|f\|_{C^{(t)}}+\sum_{|\alpha|=[t]} \sup _{x \neq y} \frac{\left|D^{\alpha} f(x)-D^{\alpha} f(y)\right|}{|x-y|^{\{t\}}} .
$$

iii) Seja $\Omega \subset R$ um domínio $C^{\infty}$, se $t \geq 0$ então $\widetilde{C^{t}}(\Omega)$ denota a restrição de $C^{t}\left(R^{n}\right)$ a $\Omega$.

Teorema 4.2.6 Seja $\Omega \subset R^{n}$ um domínio arbitrário, $1<p<\infty, t \geq 0 e$ $s>t+\frac{n}{p}$. Então

$$
H_{p}^{s}(\Omega) \subset \bar{C}^{t}(\Omega)
$$

Teorema 4.2.7 Sejam $\Omega \subset R^{n}$ um domínio arbitrário, $1<p<\infty, t \geq 0$ e $s>1+\frac{1}{p}$ então a aplicação $\gamma(f)=\left.f\right|_{\partial \Omega}$ é uma aplicação linear contínua de $H_{p}^{s}(\Omega)$ sobre $W^{s-\frac{1}{p}, p}(\partial \Omega) \subset L^{p}(\partial \Omega)$ tal que existe uma aplicação linear contínua $\bar{\gamma}$ com $\gamma \circ \bar{\gamma}=I$, a aplicação identidade de $W^{s-\frac{1}{p}, p}(\partial \Omega)$ em si mesmo.

Agora definamos nossos espaços tendo em mente as condições de fronteira. Primeiro definamos as condições de fronteira admissíveis.

Definição 4.2.5 Seja $\Omega \subset R$ um domínio $C^{\infty}$. Além disso, seja

$$
B_{j} f(x)=\sum_{|\alpha| \leq m_{j}} b_{j, \alpha}(x) D^{\alpha} f, \quad b_{j, \alpha}(x) \in C^{\infty}(\partial \Omega),
$$

$j=1, \ldots, k$, operadores diferenciáveis em $\partial \Omega$. Então $\left\{B_{j}\right\}_{j=1}^{k}$ se chama um sistema normal se

$$
0 \leq m_{1}<m_{2}<\cdots<m_{k}
$$

e se, para qualquer vetor normal $v_{x}$ com respeito a $\partial \Omega$ em um ponto $x \in \partial \Omega$, se verifica

$$
\sum_{|\alpha|=m,} b_{j, \alpha} v_{x} \neq 0, \quad j=1, \ldots, k
$$


Definição 4.2.6 Seja $\Omega \subset R^{n} u m$ domínio $C^{\infty}$. Além disso, seja $\left\{B_{j}\right\}_{j=1}^{k}$ um sistema normal.

Para $s>0$ e $1<p<\infty$ temos

i) $S e s-\frac{1}{p}<m_{1}$, então $H_{p,\left\{B_{j}\right\}}^{s}(\Omega)=H_{p}^{s}(\Omega)$.

ii) Se $m_{l}<s-\frac{1}{p}<m_{l+1}$, então

$$
H_{p,\left\{B_{j}\right\}}^{s}(\Omega)=\left\{f: f \in H_{p}^{s}(\Omega),\left.B_{j} f\right|_{\partial \Omega}=0 \text { para } j \leq l\right\} .
$$

Observação 4.2.2 Em nosso caso, isto é, em (4.1) temos que $k=1 e$ $m_{1}=1, B_{1}=\frac{\partial u}{\partial n}$.

A definição anterior leva em conta as condições na fronteira somente quando estas fazem sentido, isto é, quando as $m_{j}$ derivadas tenham traço.

\subsection{Teoria de Interpolação e Domínios}

Agora resumiremos alguns dos resultados da teoria de interpolação aplicada aos espaços de Lebesgue definidos antes, e os relacionaremos com os domínios de definição das potências fracionárias de operadores positivos, para que possamos estabelecer a equação abstrata associada a (4.1).

Sejam $X_{1} \subset X_{0}$ espaços de Banach, com injeção contínua,

$$
S=\{z \in C: 0 \leq \operatorname{Re}(z) \leq 1\}
$$

e $S^{0}$ o interior de $S$. Seja $H\left(X_{0}, X_{1}\right)$ o conjunto de todas as funções limitadas contínuas $F: S \rightarrow X_{0}$, que são holomorfas em $S^{0}$, tais que a seguinte norma é finita:

$$
\|F\|=\max _{j} \sup _{y}\|F(j+i y)\|_{j}
$$

onde $j=0,1,-\infty<y<\infty$, e $\|\cdot\|_{j}$ é a norma em $\mathrm{X}_{j}$. Para $0 \leq \theta \leq 1$, o espaço de interpolação $X_{\theta}$ é definido por

$$
X_{\theta}=\left[X_{0}, X_{1}\right]_{\theta}=\left\{F(\theta): f \in H\left(X_{0}, X_{1}\right)\right\},
$$

com a norma $\|f\|_{\theta}=\inf \{\|F\|: F(\theta)=f\}$.

Os seguintes fatos podem ser provados:

i) $X_{1}$ é densamente e continuamente imerso em $X_{\theta}$, e $X_{\theta}$ em $X_{0}$,

ii) Se $\theta_{1} \leq \theta \leq \theta_{2}$, então $\left[X_{0}, X_{1}\right]_{\theta}=\left[X_{\theta_{1}}, X_{\theta_{2}}\right]_{s}$, onde $\theta=\theta_{1}+s\left(\theta_{2}-\theta_{1}\right)$.

iii) Seja $Y_{1} \subset Y_{0}^{\prime}$ uma injeção contínua de espaços de Banach e seja $A$ : 
$X \rightarrow Y$ uma aplicação linear tal que $\|A x\|_{0} \leq C_{0}\|x\|_{0}$ e $\|A x\|_{1} \leq C_{1}$. Então $\|A x\|_{\theta} \leq C_{\theta}\|x\|_{\theta}, C_{\theta}=C_{0}^{1-\theta} C_{1}^{\theta}$.

Com o anterior, temos a seguinte propriedade de dualidade.

Teorema 4.3.1 Se um dos seguintes espaços $X_{0}$ ou $X_{1}$ é reflexivo, então

$$
\left[X_{0}, X_{1}\right]_{\theta}^{\prime}=\left[X_{\theta_{1}}^{\prime}, X_{\theta_{2}}^{\prime}\right]_{\theta} \quad 0<\theta<1 .
$$

Agora apresentaremos as fórmulas de interpolação para os espaços de Lebesgue.

Teorema 4.3.2 Sejam $1<p_{0}, p_{1}<\infty, \quad-\infty<s_{0}, s_{1}<\infty$ e $0<\theta<1$. Então

$$
\left[H_{p_{0}}^{s_{0}}\left(R^{n}\right), H_{p_{1}}^{s_{1}}\left(R^{n}\right)\right]_{\theta}=H_{p}^{s}\left(R^{n}\right)
$$

onde $s=(1-\theta) s_{0}+\theta s_{1}$, e $\frac{1}{p}=\frac{1-\theta}{p_{0}}+\frac{\theta}{p_{1}}$.

Teorema 4.3.3 Seja $\Omega \subset R^{n}$ um dominio $C^{\infty}$ limitado. Sejam $1<p_{0}, p_{1}<$ $\infty,-\infty<s_{0}, s_{1}<\infty$ e $0<\theta<1$. Então

$$
\left[H_{p_{0}}^{s_{0}}(\Omega), H_{p_{1}}^{s_{1}}(\Omega)\right]_{\theta}=H_{p}^{s}(\Omega)
$$

onde $s=(1-\theta) s_{0}+\theta s_{1}, e \frac{1}{p}=\frac{1-\theta}{p_{0}}+\frac{\theta}{p_{1}}$.

Teorema 4.3.4 Seja $\Omega \subset R$ um domínio $C^{\infty}$ limitado. Além disso, seja $\{B\}_{j=1}^{k}$ um sistema normal de condições de fronteira. Sejam $m$ um número natural tal que $m>m_{k}, 1<p<\infty$ e $0<\theta<1$.

i) Se não existe um número $m_{j}$ tal que $m \theta-\frac{1}{p}=m_{j}$, então

$$
\left[L^{p}(\Omega), H_{p,\left\{B_{j}\right\}}^{m}(\Omega)\right]_{\theta}=H_{p,\left\{B_{j}\right\}}^{m \theta}(\Omega)
$$

ii)Seja $m \theta-\frac{1}{p}=m_{l}$. Suponha que os coeficientes $b_{l, \alpha}(x)$ e suas primeiras derivadas são contínuas em $\Omega$, então

$$
\left[L^{p}(\Omega), H_{p,\left\{B_{j}\right\}}^{m}(\Omega)\right]_{\theta}=\left\{f: f \in H_{p,\left\{B_{j}\right\}}^{m}(\Omega), B_{j} f \in H_{p,\left\{B_{j}\right\}}^{\frac{1}{p}(\Omega)}\right\} .
$$


Agora estabeleceremos um resultado que conecta estes espaços de interpolação com os domínios de potências fracionárias de operadores positivos.

Teorema 4.3.5 Seja $\Lambda$ um operador positivo. Suponhamos que existem dois números positivos $\epsilon$ e $C$ tais que $\Lambda^{i t}$ é um operador limitado para $-\epsilon \leq t \leq \epsilon$ e $\left\|\Lambda^{i t}\right\| \leq C$. Se $\alpha$ e $\beta$ são dois números complexos , $0 \leq \operatorname{Re} \alpha<\operatorname{Re} \beta<\infty$ e $0<\theta<1$, então

$$
\left[D\left(\Lambda^{\alpha}\right), D\left(\Lambda^{\beta}\right)\right]_{\theta}=D\left(\Lambda^{\alpha(1-\theta)+\theta \beta}\right) .
$$

O teorema anterior nos dá uma relação entre os espaços fracionários (não conhecidos), obtidos por meio da interpolação de outros dois (conhecidos).

\subsection{Potência fracionária negativa de um espaço.}

Agora queremos definir a potência fracionária de um espaço com relação ao operador definido por (4.1), incluindo as potências negativas. Comecemos definindo o operador.

Consideramos $A=\operatorname{diag}\left(A_{1}, A_{2}, \ldots \ldots, A_{n}\right)$ em $L^{p}\left(\Omega, C^{n}\right)$, o operador definido por

$$
\left\{\begin{array}{l}
D\left(A_{i}\right)=H_{p,\{B\}}^{2}(\Omega) \\
A_{i} u=-\operatorname{Div}\left(a_{i} \nabla u\right)+\sum_{j=1}^{n} b_{j}^{i}(x) \frac{\partial u}{\partial x_{j}}+\lambda u
\end{array}\right.
$$

onde $B$ é o operador de fronteira $B u=\frac{\partial u}{\partial n_{\alpha}}$. Seja $A^{\prime}=\operatorname{diag}\left(A_{1}^{\prime}, A_{2}^{\prime}, \ldots \ldots, A_{n}^{\prime}\right)$ o operador dual em $L^{q}\left(\Omega, C^{n}\right)$, onde $\frac{1}{p}+\frac{1}{q}=1$, definido por

$$
\left\{\begin{array}{l}
D\left(A_{i}^{\prime}\right)=H_{q,\{C\}}^{2}(\Omega) \\
A_{i}^{\prime} v=-\operatorname{Div}\left(a_{i} \nabla v\right)+\operatorname{Div}\left(v B_{i}\right)+\lambda v
\end{array}\right.
$$

onde $C$ é o operador de fronteira $C v=\frac{\partial v}{\partial n_{\alpha}}+v B . n$.

Agora temos os seguintes resultados:

i) $A^{\prime}$ é um isomorfismo de $H_{q,\{C\}}^{2}(\Omega)$ sobre $L^{q}\left(\Omega, C^{n}\right)$.

ii) Denotamos por $A^{\prime \prime}$ o operador dual de $A^{\prime}$. Então $A^{\prime \prime}$ é um isomorfismo de $L^{p}\left(\Omega, C^{n}\right)$ sobre $\left(H_{q,\{C\}}^{2}(\Omega)\right)^{\prime}$.

iii) $A^{\prime \prime} \cong A$ em $H_{p,\{B\}}^{2}(\Omega)$.

Com isto definimos o operador $A_{-1}$ em $\left(H_{q,\{C\}}^{2}\left(\Omega, C^{n}\right)\right)^{\prime}$ por

$$
\left\{\begin{array}{l}
D\left(A_{-1}\right)=L^{p}\left(\Omega, C^{n}\right) \\
A_{-1} u=A^{\prime \prime} u \quad \text { para todo } u \in L^{p}\left(\Omega, C^{n}\right) .
\end{array}\right.
$$


Portanto, temos o seguinte diagrama

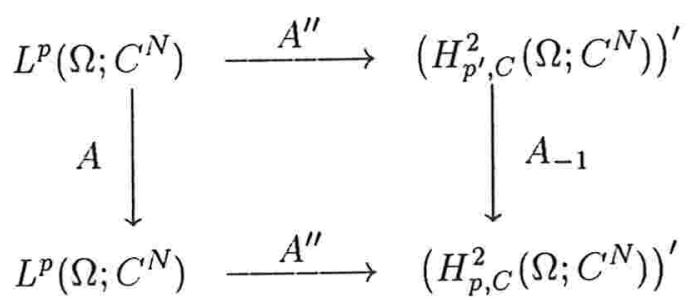

Como $A_{-1} \cong A^{\prime \prime} \circ A \circ\left(A^{\prime \prime}\right)^{-1}$ em um subespaço denso de $\left(H_{q,\{C\}}^{2}\left(\Omega, C^{n}\right)\right)^{\prime}$ (por exemplo $\mathrm{C}_{0}^{\infty}$ ) e são operadores fechados, temos que $A_{-1} \cong A^{\prime \prime} \circ A \circ\left(A^{\prime \prime}\right)$. Com isto temos o seguinte resultado.

Proposição 4.4.1 Seja $A_{-1}$ um operador setorial com $\rho(A)=\rho\left(A_{-1}\right)$. Além disso, dado $\theta>0$, definimos

$$
X_{-1}^{\theta}=D\left(A_{-1}^{\theta}\right)
$$

então $A_{-1}$ é um operador setorial em $X_{-1}^{\theta}$ que denotaremos por $A_{\theta-1}$.

\section{Prova:}

Do diagrama anterior e do fato que $A^{\prime \prime}$ é um isomorfismo temos a seguinte identidade:

$$
\left(A_{-1}-\mu I\right)^{-1}=\left(A^{\prime \prime}(A-\mu I)\left(A^{\prime \prime}\right)^{-1}\right)^{-1},
$$

$\operatorname{logo} \rho(A)=\rho\left(A_{-1}\right)=\rho\left(A_{\theta-1}\right)$,

Para finalizar a prova observamos que se $u \in X_{-1}^{\theta}$ então

$$
\begin{aligned}
\left\|\left(A_{\theta-1}-\mu I\right)^{-1} u\right\|_{X_{-1}^{\theta}} & =\left\|A_{-1}^{\theta}\left(A_{\theta-1}-\mu I\right)^{-1} u\right\|_{X_{-1}} \\
& =\left\|\left(A_{\theta-1}-\mu I\right)^{-1} A_{-1}^{\theta} u\right\|_{X_{-1}^{\theta}} \\
& =\left\|\left(A_{\theta-1}-\mu I\right)^{-1} A_{-1} u\right\|_{X_{-1}^{\theta}} \\
& =\left\|\left(A^{\prime \prime}(A-\mu I)^{-1}\left(A^{\prime \prime}\right)^{-1}\right) A_{-1}^{\theta} u\right\|_{X_{-1}} \\
& \leq\left\|A^{\prime \prime}(A-\mu I)^{-1}\right\|_{L\left(L^{p}, X_{-1}\right)}\left\|\left(A^{\prime \prime}\right)^{-1} A_{-1}^{\theta} u\right\|_{L^{p}} \\
& \leq\left\|A^{\prime \prime}\right\|_{L\left(L^{p}, X_{-1}\right)}\left\|(A-\mu I)^{-1}\right\|_{L^{p}}\left\|\left(A^{\prime \prime}\right)^{-1}\right\|_{L\left(X, L^{p}\right)}\left\|A_{-1}^{\theta} u\right\|_{X_{-1}} \\
& \leq C\left\|(A-\mu I)^{-1}\right\|_{L^{p}}\left\|A_{-1}^{\theta} u\right\|_{X_{-1}}<\frac{M}{\mu-a}\left\|A_{-1}^{\theta} u\right\|_{X_{-1}} \\
& =\frac{M}{\mu-a}\|u\|_{X_{-1}^{\theta}} .
\end{aligned}
$$


Notação: Denotamos por $X_{\theta}=D\left(A^{\theta}\right)$.

Agora temos o seguinte resultado:

Teorema 4.4.1 Se $0 \leq \theta \leq 1$, então $X_{\theta}=H_{p,\{B\}}^{2}(\Omega)=X_{-1}^{\theta+1}$.

Prova:

Primeiro provemos que $X_{-1}^{2}=X^{1}$. De fato,

$$
\begin{aligned}
X_{-1}^{2} & =\left\{u \in X_{-1}: A_{-1}^{2} u \in X_{-1}\right\} \\
& =\left\{u \in X_{-1}: A_{-1} \circ A_{-1} u \in X_{-1}\right\} \\
& =\left\{u \in X_{-1}: A_{-1} u \in L^{p}\left(\Omega, C^{n}\right)\right\} \\
& =\left\{u \in L^{p}\left(\Omega, C^{n}\right): A_{-1} u \in L^{p}\left(\Omega, C^{n}\right)\right\} \\
& =\left\{u \in L^{p}\left(\Omega, C^{n}\right): A u \in L^{p}\left(\Omega, C^{n}\right)\right\} \\
& =D(A) \\
& =X^{1} .
\end{aligned}
$$

Então podemos escrever

$$
D\left(A^{\theta}\right)=\left[D\left(A^{0}\right), D\left(A^{1}\right)\right]_{\theta}=\left[D\left(A_{-1}^{1}\right), D\left(A_{-1}^{2}\right)\right]_{\theta}=D\left(A_{-1}^{\theta+1}\right) .
$$

Para terminar a prova, observamos que

$$
D\left(A^{\theta}\right)=\left[L^{p}\left(\Omega, C^{n}\right), H_{p,\{B\}}^{2}\left(\Omega, C^{n}\right)\right]_{\theta}=H_{p,\{B\}}^{2 \theta}\left(\Omega, C^{n}\right) .
$$

\#

Observação 4.4.1 Tendo estes resultados em mente, definimos, para todo $0 \leq s \leq 1$,

$$
X^{-s}=X_{-1}^{1-s}
$$

\subsection{Hipóteses}

Nesta seção fixaremos as hipóteses que usaremos ao longo da tese. A hipótese seguinte é usada para garantir a existência e unicidade da solução da equação abstrata relacionada com (4.1).

(H1) $f=\left(f_{1}, \ldots \ldots ., f_{N}\right)$, de $R^{N}$ em $R^{N}$, localmente Lipschitz em conjuntos limitados, e $g=\left(g_{1}, \ldots \ldots . ., g_{N}\right) \in C^{2}\left(R^{N}, R^{N}\right)$ são tais que existem 
constantes $c_{i}^{0}$ e $d_{i}^{0}$ satisfazendo a seguinte condição

$$
\begin{aligned}
& \lim \inf _{|s| \rightarrow \infty} \frac{f_{i}(s)}{s_{i}} \leq c_{i}^{0} \\
& \lim \inf _{|s| \rightarrow \infty} \frac{g_{i}(s)}{s_{i}} \leq d_{i}^{0}
\end{aligned}
$$

A hipótese seguinte é para garantir a existência do atrator global.

(H2) Supondo que $(H 1)$ se verifica, suporemos que $c_{i}^{0}$ e $d_{i}^{0}$ são tais que o primeiro autovalor do seguinte problema é positivo:

$$
\left\{\begin{array}{c}
-\operatorname{Div}\left(a \nabla u_{i}\right)+\sum_{j=1}^{n} B_{j}(x) \frac{\partial u_{i}}{\partial x_{j}}+\lambda u_{i}-c_{i}^{0} u_{i}=\mu_{i} u_{i} \quad \text { em } \Omega \\
\frac{\partial v_{i}}{\partial n_{\alpha}}=d_{0} u_{i}
\end{array}\right.
$$

\subsection{Existência local}

Segue da proposição (4.4.1) e dos resultados de Henry [Teorema (1.2.1) em [11] ou Teorema 1.3.1] que $A_{-\beta}$ gera um semigrupo analítico em $X^{-\beta}$, para $-\beta<\alpha<1-\beta$,

$$
\begin{gathered}
\left\|e^{-A_{-\beta} t} u_{0}\right\|_{X^{\alpha}} \leq M e^{-\varepsilon t}\left\|u_{0}\right\|_{X^{\beta}}, t \geq 0 \\
\left\|e^{-A_{-\beta} t} u_{0}\right\|_{X^{\alpha}} \leq M e^{-\varepsilon t} t^{-(\alpha+\beta)}\left\|u_{0}\right\|_{X^{\beta}} t>0,
\end{gathered}
$$

para algum $\varepsilon>0, M>0$. Em particular, se $B_{j}=0, \quad j=1 \ldots . . n, \lambda$ pode ser qualquer número positivo.

Nós queremos escolher $\alpha, \beta$ e $p$ de maneira que

i) $X^{\alpha} \subset \overline{C^{0}}(\Omega)$;

ii) $X^{1-\beta}=H_{p}^{2(1-\beta)}(\Omega)$, em outras palavras, $X^{1-\beta}$ não incorpora as condições de fronteira;

iii) $\alpha+\beta<1$.

Portanto tomaremos $\mathrm{p}, \alpha$ e $\beta$ satisfazendo

$$
\frac{n}{2 p}<\alpha<1-\beta<1-\frac{1}{2 q}=\frac{1}{2}+\frac{1}{2 p} .
$$

Note que

- i) segue dos teoremas (4.4.1) e (4.2.6),

- ii) segue da definição (4.2.6), teorema (4.4.1), e do teorema (4.2.5). 
É fácil ver que (4.4) pode ser verificada se $p$ é suficientemente grande ( por exemplo, se $p=n$ ).

Corolário 4.6.1 Se $p$, $\alpha$ e $\beta$ satisfazem (4.4), então

$$
X^{\alpha}=H_{p}^{2 \alpha}(\Omega) \quad \text { e } \quad X^{-\beta}=\left(H_{q}^{2 \beta}(\Omega)\right)^{\prime}
$$

Definição 4.6.1 Consideremos a aplicação $g_{\gamma}: X^{\alpha} \rightarrow X^{-\beta}$ definida por

$$
\left\langle g_{\gamma}(u), \phi\right\rangle=\int_{\partial \Omega} \gamma(g(u)) \gamma(\phi), \quad \text { para toda } \phi \in H_{q}^{2 \beta}(\Omega)
$$

onde $\gamma$ denota o operador traço.

Similarmente definimos $f_{\Omega}: X^{\alpha} \rightarrow X^{-\beta}$ por

$$
\left\langle f_{\gamma}(u), \phi\right\rangle=\int_{\Omega}\left(f(u)(\phi), \quad \text { para toda } \phi \in H_{q}^{2 \beta}(\Omega)\right.
$$

e por $h=f_{\Omega}+g_{\gamma}$.

É fácil ver que $f_{\Omega}$ e $g_{\gamma}$ estão bem definidas.

Teorema 4.6.1 Suponhamos que (H1) se verifica e que $\alpha, \beta$ e p satisfazem (4.4), então h é Lipschitz em conjuntos limitados de $X^{\alpha}$.

Prova: Sejam u,v $\in V \subset X^{\alpha}$, onde $V$ é limitado .. Então temos que

$$
\left\|g_{\gamma}(u)-g_{\gamma}(v)\right\|_{X^{-\beta}}=\sup _{\phi \in H_{q}^{2 \beta}(\Omega),\|\phi\|=1}\left\|\left\langle g_{\gamma}(u)-g_{\gamma}(v), \phi\right\rangle\right\|,
$$

$\mathrm{e}$

$$
\begin{gathered}
\left\|\left\langle g_{\gamma}(u)-g_{\gamma}(v), \phi\right\rangle\right\| \leq \int_{\partial \Omega}\left|\left(g_{\gamma}(u)-g_{\gamma}(v)\right) \gamma(\phi)\right| \\
\leq\|\gamma(g(u)-g(v))\|_{L^{p}(\Omega)}\|\gamma(\phi)\|_{L^{q}(\partial \Omega)} \\
\leq K^{\prime}\|u-v\|_{X^{\alpha}}\|\phi\|_{H_{q}^{2 \beta}(\Omega)} .
\end{gathered}
$$

Similarmente provamos que $f_{\Omega}$ e Lipschitz.

Com o anterior podemos verificar o seguinte. 
Teorema 4.6.2 Suponhamos que (H1) se verifica e que $\alpha, \beta$ e p satisfazem (4.4). Então o problema abstrato parabólico

$$
\left\{\begin{aligned}
\frac{d u}{d t}+A_{-\beta} u & =h(u) \\
u(0)=u_{0} & \in X^{\alpha}
\end{aligned}\right.
$$

tem uma única solução, para qualquer $u_{0} \in X^{\alpha}$, que é dada pela fórmula da variação das constantes

$$
T(t) u_{0}=e^{-A_{-\beta} t} u_{0}+\int_{0}^{t} e^{-A_{-\beta}(t-s)} h\left(T(s) u_{0}\right) d s .
$$

Prova: O resultado segue do teorema (2.1.1) que pode ser encontrado em [11], pois pelo teorema (4.6.1), $h: X^{\alpha} \rightarrow X^{-\beta}$ é Lipschitz contínua em conjuntos limitados de $X^{\alpha}$.

Sabemos como verificar a estabilidade no problema abstrato, portanto o teorema anterior será de vital importância para estudar a estabilidade do problema original.

\subsection{Resultados de Regularidade}

Nesta seção mostraremos que a solução dada pelo teorema anterior está em $\bar{C}^{2+\epsilon}(\bar{\Omega})$, para $\varepsilon>0$, e qualquer $t>0$.

Para isto devemos, além de impor que $f$ satisfaça (H1) pedir que $f \in$ $C^{1}\left(R^{N}, R^{n}\right)$.

Teorema 4.7.1 Suponhamos que $(H 1)$ e $f \in C^{1}\left(R^{N}, R^{N}\right)$ se verifica e que $\alpha, \beta$ e p satisfazem (4.4). Sejam $u_{0} \in X^{\alpha}$ e u uma solução de (4.5). Então existe $\varepsilon>0$ tal que $u(t,.) \in \bar{C}^{2+\varepsilon}(\bar{\Omega})$, para qualquer $t>0$. Além disso, $u(t,$.$) é uma solução clássica de (4.1), para todo t>0$.

O teorema anterior mostra que a solução do problema abstrato é também a solução clássica da equação em derivadas parciais, depois de um tempo $t>0$.

Observação 4.7.1 Daqui para frente suporemos sempre que $(H 1),(H 2) e$ $f \in C^{1}\left(R^{N}, R^{N}\right)$ se verificam e que $\alpha, \beta$ e $p$ satisfazem (4.4). 


\subsection{Limitação do Semigrupo}

Nesta seção provaremos que as soluções de (4.1) com dado inicial em $X^{\alpha}$, estão definidas globalmente e órbitas de conjuntos limitados de $X^{\alpha}$ serão limitadas em $X^{\alpha}$ subconjuntos de $X^{\alpha}$. Para tanto suporemos sempre que $(H 1),(H 2)$ e $f \in C^{1}\left(R^{N}, R^{N}\right)$ se verificam e que $\alpha, \beta$ e $p$ satisfazem (4.4).

Para conseguir isto, usaremos resultados de comparação.

Definição 4.8.1 Sejam $u_{0} \in W^{2+\varepsilon, 2}(\Omega), T>0 e \bar{u}: \Omega \subset R^{n} \rightarrow R(\underline{u}$ respectivamente) uma função contínua em $[0, T] \times \bar{\Omega}$, continuamente diferenciável em $t$ e duas vezes continuamente diferenciável em $x$, para $(t, x) \in$ $(0, T) \times \Omega$. Então $\bar{u}(\underline{u}$ respectivamente) é uma super-solução (sub-solução) do problema

$$
\left\{\begin{array}{c}
u_{t}=\operatorname{Div}(a \nabla u)+\sum_{j=1}^{n} B_{j}(x) \frac{\partial u}{\partial x_{j}}+\lambda u-c_{i}^{0} u=\mu_{i} u \quad \text { em } \Omega \\
\frac{\partial v_{i}}{\partial n_{\alpha}}=g(u) \quad \text { em } \partial \Omega \\
u(0)=u_{0}
\end{array}\right.
$$

se satisfaz

$$
\left\{\begin{array}{c}
u_{t} \geq \operatorname{Div}(a \nabla u)+\sum_{j=1}^{n} B_{j}(x) \frac{\partial u}{\partial x_{j}}-\lambda u+f(u) \quad \text { em }(0, T] \times \Omega \\
\frac{\partial v_{i}}{\partial n_{\alpha}} \geq g(u) \quad e m(0, T] \times \partial \Omega \\
u(0) \geq u_{0}
\end{array}\right.
$$

(e, respectivamente, com sinal de $\geq$ substituindo o sinal $\leq$ ).

Teorema 4.8.1 (Pao [9]) Se fé localmente Lipchitz, $\bar{u}$ e u são respectivamente as super-solução e sub-solução do problema (4.7), satisfazendo

$$
\underline{u} \leq \bar{u} \quad \text { em } \quad(0, T] \times \Omega
$$

então, existe uma solução u de (4.7) tal que

$$
\underline{u} \leq u \leq \bar{u} \quad \text { em } \quad(0, T] \times \Omega .
$$

Seja $\varphi$ a primeira autofunção normalizada do problema (4.3) e $m=$ $\min _{x \in \Omega} \varphi(x)$. Sabemos que $m>0$. Para cada $\theta \in R^{+}$definimos:

$$
\sum_{\theta}=\left\{u \in X^{\alpha}:|u(x)| \leq \theta \varphi(x), \text { para todo } x \in \Omega\right\}
$$


Das hipóteses de dissipação em $f$ e $g$, sabemos que existe $\xi>0$, tal que

$$
\frac{f_{i}(s)}{s_{i}} \leq c_{i}^{0} \quad e \quad \frac{g_{i}(s)}{s_{i}} \leq d_{i}^{0}
$$

para todo $s \operatorname{com}|s| \geq \xi$.

Lema 4.8.1 Se $\theta m \geq \xi$ então $\sum_{\theta}$ é positivamente invariante para soluções locais de (4.1).

\section{Prova:}

Seja

$$
\begin{aligned}
& \tilde{\sum_{\theta}}=\left\{u \in X^{\alpha}: u(x) \leq \theta \varphi(x), \text { para todo } x \in \Omega\right\} \\
& \widehat{\sum_{\theta}}=\left\{u \in X^{\alpha}: u(x) \geq-\theta \varphi(x), \text { para todo } x \in \Omega\right\}
\end{aligned}
$$

Como

$$
\sum_{\theta}=\tilde{\sum_{\theta}} \widehat{\cap \sum_{\theta}},
$$

é suficiente provar que $\sum_{\theta}^{\sim}$ e $\widehat{\sum_{\theta}}$ são positivamente invariantes.

Seja $u_{0} \in \sum_{\theta}^{\tilde{\theta}}$, e suponhamos por contradição que existe $t_{0} \in\left[0, t_{\max }\right]$ e $x_{0} \in \bar{\Omega}$ tal que

$$
T\left(t_{0}\right) u\left(x_{0}\right)>\theta \varphi\left(x_{o}\right) .
$$

Consideremos

$$
\begin{aligned}
\bar{v}(t) & =\operatorname{Div}(a \nabla \bar{v})+\sum_{j=1}^{n} B_{j}(x) \frac{\partial \bar{v}}{\partial x_{j}}-\lambda \bar{v}+c_{0} \bar{v} \\
& \geq \operatorname{Div}(a \nabla \bar{v})+\sum_{j=1}^{n} B_{j}(x) \frac{\partial \bar{v}}{\partial x_{j}}-\lambda \bar{v}+f(\bar{v}) \\
\frac{\partial \bar{v}}{\partial n_{\alpha}} & =d_{0}(\bar{v}) \geq g(\bar{v})
\end{aligned}
$$

para todo $t \in\left(0, t_{0}\right]$.

Então $v$ é super solução do problema (4.1). Segue do teorema (4.8.1) que

$$
T(t) u(x) \leq \bar{v}(t) \text { em } \bar{\Omega}, \text { para todo } t \in\left(0, t_{0}\right] .
$$


Em particular, $T\left(t_{0}\right) u\left(x_{0}\right) \leq \theta \varphi\left(x_{o}\right)$, que é uma contradição.

Para provar que $\widehat{\sum_{\theta}}$ é positivamente invariante procede-se de maneira similar, usando agora que $\bar{v}=-\underline{v}$ como sub-solução do problema (4.1).

Lema 4.8.2 Se $V$ é um subconjunto limitado de $X^{\alpha}$ então $\bigcup_{t \geq 0} T(t) V$ é um conjunto limitado de $X^{\alpha}$.

Com o anterior provamos que as soluções de (4.1) com dado inicial em $X^{\alpha}$, estão globalmente definidas e as órbitas de conjuntos limitados de $X^{\alpha}$, são também trasformadas pelo fluxo definido por (4.1) em conjuntos limitados.

\subsection{Existência de Atratores Globais.}

Lema 4.9.1 Suponhamos $\bar{\theta} \in R$, satisfaz $\bar{\theta} m>\xi$. Entäo, para qualquer $\theta$ existe um $t_{0}$ tal que

$$
T(t) \sum_{\theta} \subset \sum_{\bar{\theta}}
$$

para todo $t \geq t_{0}$.

Prova: Seja $u \in \sum_{\theta}$. Podemos supor, sem perda de generalidade que $\theta \geq$ $\bar{\theta}$.

Seja $\bar{v}=e^{t \mu} \theta \varphi, \bar{v}=-\underline{v}$. Como no Lema (4.8.1), pode-se provar que $\bar{v}, \underline{v}$ são super e sub-soluções, respectivamente. Usando o teorema de comparação (4.8.1), temos que

$$
\bar{v} \leq T(t) u \leq \underline{v},
$$

quando $e^{t \mu} \theta \geq \theta$. Portanto $T(t) u$ intercepta $\sum_{\theta}$. Como $\sum_{\theta}$ é positivamente invariante, temos o resultado desejado.

Teorema 4.9.1 O problema (4.1) possui um atrator global $\mathfrak{A}$ em $X^{\alpha}$. Além disso, $\mathfrak{A} \subset \sum_{\theta}$, se $\theta m \geq \xi$.

Prova:

Pelo Lema (4.8.2), $T(t)$ leva conjuntos limitados de $X^{\alpha}$ em conjuntos limitados de $X^{\alpha}$, para qualquer $t \geq 0$, e o semigrupo regulariza as soluções, resta mostrar a dissipatividade. 
Seja $\bar{\theta} \in R$ tal que $\bar{\theta} m \geq \xi$. Se $u$ é um elemento qualquer de $X^{\alpha}$, segue da continuidade da inclusão $X^{\alpha} \hookrightarrow C^{0}(\bar{\Omega})$ que $u \in \sum_{\theta}$, para algum $\theta$, e portanto, aplicando o Lema (4.9.1) concluimos que $T(t) u \in \sum_{\bar{\theta}}$, para t suficientemente grande.

Seja $v=T\left(t_{0}\right) u \in \sum_{\bar{\theta}}$. Aplicando a formula das variações das constantes obtemos

$$
\begin{gathered}
\|T(t) v\|_{X^{\alpha}} \leq M e^{-\epsilon t}\|v\|_{X^{\alpha}} \\
+M \int_{0}^{\infty}\left(K\|\gamma(g T(s) v)\|_{\infty}|\partial \Omega|^{\frac{1}{p}}+\left\|f_{\Omega} T(s) v\right\|_{\infty}|\Omega|^{\frac{1}{p}}\right)(t-s)^{-(\alpha+\beta)} e^{-\epsilon(t-s)} d s
\end{gathered}
$$

onde $M$ e $K$ são independentes de $v$.

Observando que $T\left(t_{0}\right) v \in \sum_{\bar{\theta}}$, para $s \geq 0$ concluimos que, para t suficientemente grande,

$$
\left\|T\left(t_{0}+t\right) u\right\|_{X^{\alpha}}=\|T(t) v\|_{X^{\alpha}} \leq M
$$

$+M\left[\sup _{|\tau| \leq \bar{\theta}\|\varphi\|_{\infty}}|g(\tau)||\partial \Omega|^{\frac{1}{p}}+\sup _{|\tau| \leq \bar{\theta}\|=\varphi\|_{\infty}}|f(\tau)||\Omega|^{\frac{1}{p}}\right] \int_{0}^{\infty}(t-s)^{-(\alpha+\beta)} e^{-\epsilon(t-s)} d s$

para $t$ suficientemente grande. Então o conjunto composto pelos elementos tais que sua norma é limitada em $X^{\alpha}$ dado pelo lado direito da equação acima é um conjunto limitado, isto é $\left\|T(t) u_{0}\right\|_{X^{\alpha}}$ é limitado, portanto o conjunto definido acima atrai pontos. Isto prova a dissipação.

Além disso, como

$$
\mathfrak{A} \subset \sum_{\theta}
$$

para algum $\theta$, segue do Lema (4.9.1), tomando t grande, que

$$
\mathfrak{A} \subset T(t) \mathfrak{A} \subset \sum_{\bar{\theta}}
$$

se $\bar{\theta}>\xi$, portanto

$$
\mathfrak{A} \subset \sum_{\bar{\theta}}=\bigcap_{\bar{\theta} m \geq \xi} \sum_{\bar{\theta}},
$$

o que prova a segunda parte da tese. 


\section{Capítulo 5}

\section{Linearização e Estabilidade}

\subsection{A Diferencial de Frechet de uma Função}

Nesta seção queremos determinar qual é o problema linearizado do problema abstrato (4.5). Para isto necessitamos dos conceitos seguintes.

Definição 5.1.1 Sejam $X$ e $Y$ espaços de Banach, uma função $f: A \rightarrow Y$ de um conjunto $A \subset X$, se diz Frechet diferenciável em $x_{0}$, se $x_{0}$ é um ponto interior de $A$ e existe $T \in L(X, Y)$ tal que

$$
0=\lim _{x \rightarrow x_{0}} \frac{f(x)-f\left(x_{0}\right)-T(x)-T\left(x_{0}\right)}{\left\|x-x_{0}\right\|_{X}}
$$

Este limite é equivalente a afirmação que

$$
f(x)=f\left(x_{0}\right)+T\left(x-x_{0}\right)+o\left(\left\|x-x_{0}\right\|_{X}\right) \text { quando } x \rightarrow x_{0}
$$

Teorema 5.1.1 (Regra da Cadeia) Sejam $A \subset X, B \subset Y, f: A \rightarrow Y$ Frechet diferenciável em $x_{0}$ e seja $g: B \rightarrow Z$ Frechet diferenciável em $y_{0}=f\left(x_{0}\right)$. Então a função $g \circ f$ é Frechet diferenciável em $x_{0} e$

$$
d(g \circ f)\left(x_{0}\right)=d g\left(y_{0}\right) \circ d f\left(x_{0}\right)
$$

Corolário 5.1.1 Sejam $A \subset X, f: A \rightarrow Y$ Frechet diferenciável em $x_{0} e$ seja $g \in L(Y, Z)$. Então a função $g \circ f$ é diferenciável Frechet em $x_{0} e$

$$
d(g \circ f)\left(x_{0}\right)=g \circ d f\left(x_{0}\right)
$$


Relembremos que $f_{\Omega}: X^{\alpha} \rightarrow X^{-\beta}$ onde $X^{\alpha}=H_{p}^{2 \alpha}(\Omega)$, e $X^{-\beta}=$ $\left(\left(H_{q}^{2 \beta}(\Omega)\right)^{\prime}\right.$. Primeiramente queremos determinar se $f_{\Omega}$ é Frechet diferenciável e qual é sua diferencial. Para isto usaremos a seguinte notação: $D\left(f_{\Omega}\right): X^{\alpha} \rightarrow X^{-\beta}$ denota a diferencial de Frechet de $f_{\Omega}$ de $X^{\alpha}$ em $X^{-\beta}$ e $D\left(f_{u_{0}}\right)$ a diferencial de $f$, como função de $R^{N}$ em $R^{N}$, em $u_{0} \in R^{N}$.

Corolário 5.1.2 Seja $f_{\Omega}: X^{\alpha} \rightarrow X^{-\beta}$ dada por

$$
\left\langle f_{\Omega}(u), \phi\right\rangle_{\left(\left(H_{q}^{2 \beta}\right)^{\prime}(\Omega), H_{q}^{2 \beta}(\Omega)\right)}=\int_{\Omega}(f(u))(\phi), \text { para toda } \phi \in H_{q}^{2 \beta}(\Omega)
$$

Definamos

$$
\left\langle F_{\Omega, u_{0}}(u), \phi\right\rangle_{\left(\left(H_{q}^{2 \beta}\right)^{\prime}(\Omega), H_{q}^{2 \beta}(\Omega)\right)}=\int_{\Omega} D\left(f_{u_{0}}\right)(u)(\phi),
$$

para toda $\phi \in H_{q}^{2 \beta}(\Omega)$. Então

$$
D\left(f_{\Omega}\right)=F_{\Omega}
$$

Prova:

Como $f$ é $C^{1}$, temos que a diferencial de $f$ é $C^{0}(\bar{\Omega})$, por tanto aplicando a desigualdade de Hölder em (5.1), esta aplicação esta bem definida. Vejamos que $f_{\Omega}(u)$ é diferenciável Frechet em $u_{0}$, para isto temos que ver que existe uma aplicação linear $T$ tal que

$$
f_{\Omega}(u)=f_{\Omega}\left(u_{0}\right)+T_{u_{0}}\left(u-u_{0}\right)+o\left(\left\|u-u_{0}\right\|_{\alpha}\right), \text { quando } x \rightarrow x_{0} .
$$

Então

$$
\begin{aligned}
\mid\left\langle f_{\Omega}(u)\right. & \left.-f_{\Omega}\left(u_{0}\right)-F_{\Omega, u_{0}}\left(u-u_{0}\right), \phi\right\rangle_{\left(\left(H_{q}^{2 \beta}\right)^{\prime}(\Omega), H_{q}^{2 \beta}(\Omega)\right)} \mid \\
& \leq \int_{\Omega} \mid\left[\left(f(u)-f\left(u_{0}\right)-D f_{u_{0}}\left(u-u_{0}\right)\right](\phi) \mid\right. \\
& \leq\left\|f(u)-f\left(u_{0}\right)-D f_{u_{0}}\left(u-u_{0}\right)\right\|_{p}\|(\phi)\|_{q} \\
& \leq o\left(\left\|u-u_{0}\right\|_{p}\right)\|(\phi)\|_{q} \\
& \leq o\left(\left\|u-u_{0}\right\|_{\alpha}\right)\|(\phi)\|_{q}
\end{aligned}
$$

Pois $f$ é $C^{1}$. 
Agora temos $g_{\gamma}: X^{\alpha} \rightarrow X^{-\beta}$ e queremos saber se é Frechet diferenciável e qual é sua diferencial.

Corolário 5.1.3 Seja $g_{\gamma}: X^{\alpha} \rightarrow X^{-\beta}$ definido por

$$
\left\langle g_{\gamma}(u), \phi\right\rangle_{\left(\left(H_{q}^{2 \beta}\right)^{\prime}(\Omega), H_{q}^{2 \beta}(\Omega)\right)}=\int_{\partial \Omega} \gamma(g(u)) \gamma(\phi),
$$

para toda $o \in H_{q}^{2 \beta}(\Omega)$, onde $\gamma$ denota o operador traço.

Definimos

$$
\langle G(u), \phi\rangle_{\left(\left(H_{q}^{2 \beta}\right)^{\prime}(\Omega), H_{q}^{2 \beta}(\Omega)\right)}=\int_{\partial \Omega} \gamma(D g)(u)(\gamma \phi),
$$

então

$$
D\left(g_{\gamma}\right)=G
$$

\section{Prova:}

Como no teorema anterior, temos que $D g_{\gamma}: X^{\alpha} \rightarrow X^{-\beta}$ e

$D g: R^{n} \rightarrow R^{n}$, como $g$ é $C^{2}$ e pelo teorema do traço (4.2.7) temos que $\gamma(g(u)) \in W^{1-\frac{1}{p}, p}(\partial \Omega)$, novamente aplicando a desigualdade de Hölder temos que $G$ está bem definida e

$$
\begin{aligned}
& \left|\left\langle g_{\gamma}(u)-g_{\gamma}\left(u_{0}\right)-G_{u_{0}}\left(u-u_{0}\right), \phi\right\rangle_{\left(\left(H_{q}^{2 \beta}(\Omega)\right)^{\prime}, H_{q}^{2 \beta}(\Omega)\right)}\right| \\
\leq & \int_{\partial \Omega}\left|\left(\gamma(g)(u)-\gamma(g)\left(u_{0}\right)-D \gamma(g)_{u_{0}}\left(u-u_{0}\right)\right) \gamma((\phi))\right| \\
\leq & \left\|\gamma\left(g(u)-g\left(u_{0}\right)\right)-\gamma D g_{u_{0}}\left(u-u_{0}\right)\right\|_{L^{p}(\partial \Omega)}\|\gamma(\phi)\|_{L^{q}(\partial \Omega)} \\
\leq & K\left\|\left(g(u)-g\left(u_{0}\right)\right)-D g_{u_{0}}\left(u-u_{0}\right)\right\|_{L^{p}(\Omega)}\|\gamma(\phi)\|_{L^{q}(\partial \Omega)} \\
\leq & K^{-1}\left\|g(u)-g\left(u_{0}\right)-D g_{u_{0}}\left(u-u_{0}\right)\right\|_{X^{\alpha}}\|\phi\|_{H_{q}^{2 \beta}(\Omega)} .
\end{aligned}
$$

Portanto,

$$
D\left(g_{\gamma}\right)=G
$$

Notação: Denotaremos por $H:=F_{\Omega}+G$.

Sabemos que o problema abstrato parabólico

$$
\left\{\begin{array}{c}
\frac{d u}{d t}+A_{-\beta} u=h(u) \\
u(0)=u_{0} \in X^{\alpha}
\end{array}\right.
$$


tem uma única solução, para qualquer $u_{0} \in X^{\alpha}$, que é dada pela fórmula da variação das constantes

$$
T(t) u_{0}=e^{-A_{-\beta} t} u_{0}+\int_{0}^{t} e^{-A_{-\beta}(t-s)} H\left(T(s) u_{0}\right) d s .
$$

e representa bem o problema (ver torema 4.7.1). Sabemos como verificar a estabilidade do problema abstrato (ver teoremas (2.2.2) e (2.2.1)), queremos ver como isto se reflete no problema original.

Teorema 5.1.2 Suponhamos que (H1) se verifica, $\alpha, \beta$ e p satisfazem (4.4) e $f \in C^{1}\left(R^{N}, R^{N}\right)$. Seja $u_{0} \in X^{\alpha}$, então o seguinte problema

$$
\left\{\begin{array}{c}
\frac{\partial u}{\partial t}=D \Delta u+\left(D_{u_{0}} f\right)(u) \\
\frac{\partial u}{\partial n}=\left(D_{u_{o}} g\right)(u)
\end{array}\right.
$$

tem o problema linearizado de (4.5) como problema abstrato associado.

\section{Prova:}

Se fixamos $u$ e consideramos o problema elíptico,

$$
\begin{gathered}
-D i v(D \Delta v)+\sum_{j=1}^{n} B_{j}(x) \frac{\partial v}{\partial x_{j}}+\lambda v=D_{u_{0}} f(u)-\frac{d u}{d t} \quad \text { em } \Omega \\
\frac{\partial v}{\partial n}=g(u) \quad \text { em } \partial \Omega .
\end{gathered}
$$

então podemos aplicar os resultados de regularidade elípticos [17] para concluir que $v \in W^{2, p}(\Omega)=H_{p}^{2}(\Omega)$.

Agora vamos provar que $v=u$.

Da fórmula de Green segue que se $w \in H_{p}^{2}(\Omega)$ e $\phi \in H_{q, C}^{2}(\Omega)$

$$
\begin{gathered}
\int_{\Omega}(A w)(x) \phi(x) d x-\int_{\Omega} w(x) A^{\prime} \phi(x) d x=\int_{\partial \Omega}\left(\frac{\partial w}{\partial n}\right)(y)(\gamma(\phi))(y) d y \\
+\int_{\partial \Omega}\left[\frac{\partial v}{\partial n}(y)+B(y) \cdot n(y)\right](\gamma(w))(y) d y \\
=\int_{\partial \Omega}[(\gamma(w))(y)(C \phi)(y) d y-[B(\phi)](\gamma(\phi))(y)] d y \\
\left.=\int_{\partial \Omega}-[B(\phi)](\gamma(\phi))(y)\right] d y .
\end{gathered}
$$


Aplicando [16] a $v$ e tendo em mente que

$$
\begin{gathered}
(A v)(x)=D_{u_{0}} f(u)-\frac{d u}{d t} \\
(C v)(x)=\left(\left(\gamma\left(D_{u_{0}} g(u)\right)\right)(y)\right.
\end{gathered}
$$

segue que $v$ satisfaz

$$
\begin{gathered}
\int_{\Omega}\left[D_{u_{0}} f(u)-\frac{d u}{d t}\right] \phi(x) d x-\int_{\Omega} v(x) A^{\prime} \phi(x) d x \\
=\int_{\partial \Omega}\left(\left(\gamma\left(D_{u_{0}} g(u)\right)\right)(y)(\gamma(\phi))(y)\right] d y
\end{gathered}
$$

como $H_{q, C}^{2}(\Omega)$ é denso em $H_{q}^{2 \beta}(\Omega)$, segue que $v$ satisfaz, em $X^{-\beta}$, a equação

$$
\left\{\begin{array}{c}
\frac{d u}{d t}+A_{-\beta} u=\left(D_{u_{0}} f+\gamma \circ D_{u_{0}} g\right) u . \\
u(0)=u_{0} \in X^{\alpha}
\end{array}\right.
$$

A importância do teorema anterior se reflete (por exemplo, para estudar a estabilidade) no fato que é mais fácil estudar o espectro, isto é, estudar os autovalores do operador linearizado, que olhar diretamente a estabilidade na equação original. 


\section{Capítulo 6}

\section{Existência do Atrator Global para O Problema Biológico}

Agora vamos fixar as hipóteses e notações que usaremos neste capítulo. Comecemos recordando a notação dos capítulos anteriores, $X^{\alpha}=H_{p}^{2 \alpha}(\Omega)$, e $X^{-\beta}=\left(\left(H_{q}^{2 \beta}(\Omega)\right)^{\prime}\right.$, onde $\Omega \in R^{l}$ é um aberto limitado além disso, estamos supondo que $\alpha, \beta$ e $p$ satisfazem

$$
\frac{l}{2 p}<\alpha<1-\beta<1-\frac{1}{2 q}=\frac{1}{2}+\frac{1}{2 p} .
$$

Neste capítulo nos concentraremos no estudo do sistema

$$
\left\{\begin{array}{c}
\frac{\partial \mathrm{v}_{i}}{\partial t}=y_{i}(\mathrm{v})+d_{i} \Delta \mathrm{v}_{i} \quad 1 \leq i \leq r \\
\frac{\partial \mathrm{v}_{i}}{\partial n}=0
\end{array}\right.
$$

onde a não linearidade $y: R^{r} \rightarrow R^{r}$ é definida por

$$
y_{i}(\mathrm{v})=\mathrm{v}_{i}\left\{\frac{(A \mathrm{v})_{i}}{V}-\frac{\mathrm{v}^{t} A \mathrm{v}}{V^{2}}\right\}
$$

com $A$, uma matriz de $r \times r$ qualquer, e $V=\mathrm{v}_{1}+\mathrm{v}_{2}+\ldots . .+\mathrm{v}_{r}$.

Esta não linearidade foi introduzida por Vickers [29] para modelar populações genéticas. Queremos aplicar as técnicas do capítulo 4 e [18], provaremos que o problema está bem posto (no sentido do capítulo 4 e [18]). Usaremos as idéias de Amann [2],[3], juntamente com a teoria abstrata de Henry [11],como desenvolvido nos artigos de Oliva e Pereira [18], e Carvalho, Oliva, Pereira e Rodriguez-Bernal [5] e parcialmente expostos nos capítulos anteriores.

Precisamos provar a existência e unicidade das soluções, existência do atrator global e por último a linearização de forma precisa, para tirar conclusões sobre estabilidade. A primeira observação a ser feita é que o modelo 
biológico proposto por Vickers [29] só faz sentido se $\mathrm{v}_{i}>0\left(\mathrm{ou} \mathrm{v}_{i} \geq 0 \mathrm{e}\right.$ alguns deles não nulos) e isto se reflete na não linearidade, já que esta possui uma singularidade quando $V=0$. A primeira dificuldade encontrada foi mostrar (mesmo sem termos de fronteira, isto é (6.1)) que se a condição inicial é positiva então a solução é positiva. Para evitar esta dificuldade, faremos uma adaptação no modelo, mudando a não-linearidade de forma a garantir esta propriedade. Para isto, dado $\epsilon>0$, considere a função corte $\zeta \in C^{\infty}\left(R, R^{+}\right)$dada por

$$
\zeta(s)\left\{\begin{array}{l}
=1, \text { se } s \geq 2 \epsilon, \\
=\exp \left\{-\frac{s-2 \epsilon}{s-\epsilon}\right\}, \text { se } \epsilon<s<2 \epsilon \\
=0, \text { se } s \leq \epsilon
\end{array}\right.
$$

Então, a nova não linearidade $Z: R^{r} \rightarrow R^{r}$, é definida, para cada $\mathrm{v}=\left(\mathrm{v}_{1}, \mathrm{v}_{2}, \ldots, \mathrm{v}_{r}\right) \in R^{r}$, da seguinte maneira

$$
Z(\mathrm{v})=\zeta\left(\mathrm{v}_{1}\right) \zeta\left(\mathrm{v}_{2}\right) \ldots \zeta\left(\mathrm{v}_{r}\right) \cdot y(\mathrm{v}) .
$$

Portanto, garantimos que $Z=\left(z_{1}, \ldots, z_{r}\right) \in C^{1}\left(R^{r}, R^{r}\right)$, e o nosso novo problema será dado por

$$
\left\{\begin{array}{c}
\frac{\partial v_{i}}{\partial t}=z_{i}(v)+d_{i} \Delta v_{i} \quad 1 \leq i \leq r \\
\frac{\partial v_{i}}{\partial n}=0
\end{array}\right.
$$

Observe que, se estamos interessados num conjunto de soluções de (6.1) tal que $\mathrm{v}_{i}>\varepsilon_{0}>0$, podemos escolher $\epsilon<\varepsilon_{0} / 2$ em (6.2) tal que $\mathrm{v}=v$ e nada se perde. Entretanto, não é verdade que as soluções de (6.3) são as mesmas que as de (6.1) e isto é um defeito deste enfoque. A grande vantagem deste enfoque é que $Z$ é localmente Lipschitz, (e ainda $C^{1}$ ) o que facilitará e muito o estudo das linearizações.

Com esta adaptação do modelo de Vickers feita, podemos generalizá-lo e pensar em controles (ou termos "forçantes") oriundos do fluxo pela fronteira, ou seja, queremos estudar, dada uma função $k_{i}$ que definiremos adiante, o problema biológico que é modelado pelas equações

$$
\left\{\begin{array}{c}
\frac{\partial v_{i}}{\partial t}=z_{i}(v)+d_{i} \Delta v_{i} \quad 1 \leq i \leq r \\
\frac{\partial v_{i}}{\partial n}=k_{i}(v)
\end{array}\right.
$$

tem atrator global.

Provaremos que o problema está bem posto (no sentido do capítulo 4 e [18]) com o qual também provamos que este tem atrator global. Usaremos as idéias de Amann [2],[3], juntamente com a teoria abstrata de Henry 
[11].como desenvolvido nos artigos de Oliva e Pereira [18], e Carvalho, Oliva, Pereira e Rodriguez-Bernal [5], parcialmente expostos nos capítulos anteriores.

\subsection{Verificação das Hipóteses}

\subsubsection{Testando as Hipóteses para a não Linearidade no Inte- rior}

Devemos verificar que existem $c_{0}^{i}$, para $i=1, \ldots, r$, tais que a hipótese (H1) da seção (4.5) é válida. Na realidade, podemos verificar que $\frac{z_{i}(v)}{v_{i}} \leq c_{0}^{i} \quad \forall v_{i}$, de fato

$$
\begin{aligned}
\frac{z_{i}(v)}{v_{i}} & =\zeta\left(v_{1}\right) \zeta\left(v_{2}\right) \ldots \zeta\left(v_{n}\right) \cdot \frac{v_{i}}{v_{i}}\left\{\frac{(A v)_{i}}{V}-\frac{v^{t} A v}{V^{2}}\right\} \\
& =\zeta\left(v_{1}\right) \zeta\left(v_{2}\right) \ldots \zeta\left(v_{n}\right) \cdot\left\{\frac{(A v)_{i}}{V}-\frac{v^{t} A v}{V^{2}}\right\} \leq \frac{\left|(A v)_{i}\right|}{\|v\|_{1}}+\frac{|\langle A v, v\rangle|}{\|v\|_{1}^{2}} \\
& \leq \frac{\|A v\|_{1}}{\|v\|_{1}}+\|A\|_{1} \leq 2\|A\|_{1} .
\end{aligned}
$$

Portanto,

$$
z_{i}(v) \leq 2\|A\|_{1} v_{i}, \quad \forall v_{i} .
$$

Pela definição, temos que $Z$ é uma função $C^{1}$ de $R^{r}$ em $R^{r}$, e portanto, como vimos anteriormente, é localmente Lipschitz em $X^{\alpha}$.

Com isto temos uma das hipóteses do teorema (2.1.1). Logo usando o Teorema (2.1.2) temos que a solução de (6.3) existe para todo $t>0$.

Também temos que construir as funções $k_{i}$ tais que $\frac{\partial v_{i}}{\partial n}=k_{i}(v) \leq-d_{0}^{i} v_{i}$.

\subsubsection{Construção da não Linearidade na Fronteira}

Consideremos um equilíbrio espacialmente constante $\bar{m} \in R^{r}$ do sistema de equações diferenciais ordinárias

$$
\frac{d p_{i}}{d t}=p_{i}\left((A p)_{i}-p^{t} A p\right) \quad 1 \leq i \leq r,
$$

onde $\sum \bar{m}_{i}=1$. Observemos primeiramente que $\bar{m}$ também é um equilíbrio do sistema (6.3) se escolhemos $2 \epsilon<\inf \bar{m}_{i}$. Nosso trabalho é controlar o comportamento das órbitas perto de $\bar{m}$, (ou seja, controlar a estabilidade de $\bar{m})$ através de um termo forçante $(k)$ atuando somente na fronteira. Queremos portanto que o termo forçante seja uma função suave e que mantenha 
$\bar{m}$ como ponto de equilíbrio do sistema (6.3), portanto dado um parâmetro $d_{0}$, consideraremos o termo forçante $k: R^{r} \rightarrow R^{r}$, definido por

$$
k_{i}(v)=-2 d_{0}\left(2-e^{-\|v-\bar{m}\|^{2}}\right)\left(v_{i}-\overline{m_{i}}\right) .
$$

Observação 6.1.1 Na realidade, matematicamente prosseguiremos ao contrário, isto é, se $\bar{v}$ é um ponto de equilíbrio espacialmente constante do sistema de reação (6.3) (ou (6.4)) e definindo $V=\sum \bar{v}_{i}$, então assumiremos que $\bar{m}=\frac{\bar{v}}{V}\left(m_{i}>0\right)$ é um ponto de equilibrio assintóticamente estável do sistema (6.6) e investigaremos sob que circunstâncias é assintoticamente estável para (6.3).

Escolheremos $d_{0}$ mais adiante. Observemos que $k$ é $C^{\infty}$ e se $\left|v_{i}\right| \geq 2 \bar{m}_{i}>$ 0 temos que

$$
\frac{k_{i}(v)}{\left(v_{i}\right)} \leq-d_{0}
$$

de fato,

1. Se $v_{i}<0$, e portanto $\frac{\bar{m}_{i}}{\left(-v_{i}\right)}>0$, então

$$
\frac{\left(v_{i}-\bar{m}_{i}\right)}{v_{i}}=\frac{\left(-v_{i}+\bar{m}_{i}\right)}{-v_{i}}=1+\frac{\bar{m}_{i}}{\left(-v_{i}\right)} \geq 1,
$$

$\log 0$

$$
-2 d_{0}\left(2-e^{-\|v-\bar{m}\|^{2}}\right) \frac{\left(v_{i}-\bar{m}_{i}\right)}{v_{i}} \leq-2 d_{0}\left(2-e^{-\|v-\bar{m}\|^{2}}\right) \leq-2 d_{0} \leq-d_{0} .
$$

2. Se $v_{i}>0$, e portanto $-\frac{\bar{m}_{i}}{v_{i}} \geq-\frac{1}{2}$, o que mostra que

$$
\frac{\left(v_{i}-\bar{m}_{i}\right)}{v_{i}}=1-\frac{\bar{m}_{i}}{v_{i}} \geq \frac{1}{2},
$$

$\log 0$

$$
-2 d_{0}\left(2-e^{-\|v-\bar{m}\|^{2}}\right) \frac{\left(v_{i}-\bar{m}_{i}\right)}{v_{i}} \leq-d_{0}\left(2-e^{-\|v-\bar{m}\|^{2}}\right) \leq-d_{0} .
$$

Resumindo temos o seguinte teorema.

Teorema 6.1.1 As não linearidades $Z$ e $k$ do problema biológico (6.4), são tais que $Z \in C^{1}\left(R^{r}, R^{r}\right), k \in C^{2}\left(R^{r}, R^{r}\right)$ e satisfazem a hipótese (H1). Mais ainda se $\alpha, \beta$ e p satisfazem (4.4) então $h=Z_{\Omega}+k_{\gamma}$ é Lipschitz em conjuntos limitados de $X^{\alpha}$. 


\subsubsection{Teste da interação entre a não linearidade da fronteira e do interior}

Já sabemos que as não-linearidades do nosso problema biológico (denotadas anteriormente por $Z$ e $k$ ), são tais que $k_{i}(v) \leq-d_{0} v_{i}$ e $z_{i}(v) \leq c_{0}^{i} v_{i}$, $Z \in C^{1}\left(R^{r}, R^{r}\right), k \in C^{2}\left(R^{r}, R^{r}\right)$, (H1) se verifica e escolhemos $\alpha, \beta$ e $p$ satisfazendo (4.4). Logo para aplicar os resultados dos capítulos 4 e 5 , precisamos mostrar que (H2) se verifica, isto é, precisamos escolher $d_{0}$ tal que o problema de autovalor (4.3) tem o primeiro autovalor positivo.

Para determinar o problema (4.3) no nosso caso, primeiramente temos que somar e subtrair $\lambda v_{i}$ a (6.4) ( $\lambda$ uma constante positiva),

$$
\left\{\begin{array}{c}
\frac{\partial v_{i}}{\partial t}=z_{i}(v)+d_{i} \Delta v_{i}-\lambda v_{i}+\lambda v_{i} \\
\leq d_{i} \Delta v_{i}-\lambda v_{i}+(\lambda+2\|A\|) v_{i}=d_{i} \Delta v_{i}-\lambda v_{i}+c_{0}^{i} v_{i} \\
\frac{\partial v_{i}}{\partial n}=k_{i}(v) \leq-d_{0} v_{i}
\end{array}\right.
$$

Agora verifiquemos que se $\|A\|$ é suficientemente pequena, então existem $c_{0}^{i}, d_{0}$ tais que o primeiro autovalor $\left(\mu_{1}\right)$ do problema (6.7)é positivo. Primeiro um lema:

Lema 6.1.3.1 Consideremos o operador linear $J$ definido por

$$
\left\{\begin{array}{c}
J(v)=-d \Delta v+\lambda v=\mu v \\
\frac{\partial v}{\partial n}=0
\end{array}\right.
$$

então temos que $\mu_{1}$, o primeiro valor próprio de $J$, é positivo.

Prova:

Observe que $\mu_{1}-\lambda=\bar{\mu}_{1}$ onde $\bar{\mu}_{1}$ é o primeiro autovalor do Laplaciano com condicão de Neumann zero, que sabemos ser zero. Portanto temos que $\mu_{1}=\lambda>0$.

Consideremos agora o problema

$$
\left\{\begin{array}{c}
-d_{i} \Delta v_{i}+\lambda v_{i}-c_{0}^{i} v_{i}=\mu_{1} v_{i} \\
\frac{\partial v_{i}}{\partial n}=-d_{0} v_{i}
\end{array}\right.
$$

Sabemos que $c_{0}^{i}=(\lambda+2\|A\|)$ e devemos verificar que existe $d_{0}$ tal que o primeiro autovalor é positivo.

Com o anterior, e com os comentários sobre a hipótese de dissipação apresentadas no capítulo 5, estamos em condições de provar o seguinte teorema. 
Teorema 6.1.2 Seja o problema

$$
\left\{\begin{array}{c}
-d \Delta u+\lambda u-c_{0} u=\mu u \\
\frac{\partial u}{\partial n}=-d_{0} u
\end{array}\right.
$$

onde $c_{0}=\lambda+2\|A\|$. Então, se $\|A\|<<1$, existe $d_{0}$ tal que o primeiro autovalor de (6.9) é positivo, em outras palavras, a hipótese (H2) é verificada.

Prova:

O problema (6.9) é o mesmo que

$$
\left\{\begin{array}{c}
-d \Delta u-2\|A\| u=\mu u \\
\frac{\partial u}{\partial n}=-d_{0} u
\end{array}\right.
$$

o qual é equivalente ao problema

$$
\left\{\begin{array}{c}
-d \Delta u=\left(\mu^{6}+2\|A\|\right) u \\
\frac{\partial u}{\partial n}=-d_{0} u
\end{array}\right.
$$

o qual é equivalente ao problema

$$
\left\{\begin{array}{c}
-d \Delta u+\bar{\lambda} u=0 \\
\frac{\partial u}{\partial n}=-d_{0} u
\end{array}\right.
$$

onde $\bar{\lambda}=-\mu-2\|A\|$. Logo, se $\|A\|<<1$, como os valores próprios do problema variam continuamente em $d_{0}$, e se $d_{0}$ é positivo, pela observação do apêndice (A.0.3), temos que o primeiro autovalor do problema (6.10) é positivo, logo o primeiro autovalor de (6.9) também é.

\subsection{Aplicação dos Resultados Abstratos}

\subsubsection{Limitação do Semigrupo, Sub e Super-solução}

O próximo passo é mostrar a existência de uma super-solução e uma subsolução para nosso problema biológico. Nosso problema é

$$
\left\{\begin{array}{c}
\frac{\partial u_{i}}{\partial t}=d_{i} \Delta u_{i}+z_{i}(u) \\
\frac{\partial u_{i}}{\partial n}=k_{i}(u)
\end{array} \quad 1 \leq i \leq r,\right.
$$

como foi visto antes $z_{i}(u) \leq 2\|A\| u_{i}$. Então

$$
\left\{\begin{array}{c}
\frac{\partial u_{i}}{\partial t}=d_{i} \Delta u_{i}+c_{0}^{i} u_{i} \geq d_{i} \Delta u_{i}+z_{i}(u) \\
\frac{\partial u_{i}}{\partial n}=-d_{0} u_{i} \geq k_{i}(u)
\end{array}\right.
$$


portanto qualquer solução do problema

$$
\left\{\begin{array}{c}
\frac{\partial u_{i}}{\partial t}=d_{i} \Delta u_{i}-\lambda u_{i}+(\lambda+2\|A\|) u_{i} \\
\frac{\partial u_{i}}{\partial n}=-d_{0} u_{i}
\end{array}\right.
$$

é super-solução do problema (6.4). Seja $\varphi$ a primeira autofunção normalizada do problema (6.12) e $m=\min _{x \in \Omega} \varphi(x)$. Sabemos que $m>0$. Para cada $\theta \in R^{+}$definimos:

$$
\sum_{\theta}=\left\{u \in X^{\alpha}:|u(x)| \leq \theta \varphi(x), \text { para todo } x \in \Omega\right\}
$$

Aplicando os resultados dos capítulos 4 e 5 temos que, pelo lema 4.8 .1 o conjunto

$$
\sum_{\theta}=\left\{u \in X^{\alpha}:|u(x)| \leq \theta \varphi(x), \text { para todo } x \in \Omega\right\}
$$

é positivamente invariante.

\subsection{Existência do Atrator Global}

Aplicando o Teorema 4.9.1 temos que o seguinte resultado

Teorema 6.3.1 O problema (6.11) tem atrator global $\mathfrak{B}$ em $X^{\alpha}$. Além disso, $\mathfrak{B} \subset \sum_{\theta}$ se $\theta m \geq \xi$. 


\section{Capítulo 7}

\section{Equilíbrios}

Nesta seção, fazendo uso das técnicas anteriores, provaremos de outra forma os resultados expostos em [29], o Teorema (2.2) de [29] é generalizado fazendo uso dos artigos [11], [18], [19] e [20]. Lembremos que na seção 6.1.1 provamos que a solução de (6.3) está definida para todo $t \geq t_{0}$ e é única. Portanto podemos falar de estabilidade assintótica. A vantagem é que nós não imporemos solução de nenhum tipo pois, ela depende do tamanho assim como da forma do domínio $\Omega$ (como feito em [29]). Nós usaremos os teoremas obtidos por D. Henry em [11] expostos aqui no capítulo 2. Para isto, devemos fazer algumas apreciações preliminares. Primeiro distinguir os distintos tipos de estabilidade segundo o exposto nos capítulos 2,3 e 4 .

\subsection{Equilíbrio Instável}

Definição 7.1.1 Diremos que um ponto de equilíbrio espacialmente constante $\bar{v}$ é temporalmente estável para a equação (6.3) se é assintoticamente estável para a equação diferencial ordinária seguinte

$$
\frac{d v_{i}}{d t}=z_{i}(v) \quad 1 \leq i \leq r
$$

isto é, da equação (6.3) com todos os $d_{i}=0$.

$O$ ponto de equilíbrio $\bar{v}$ é dito uniformemente estável se é assintoticamente estável para todos os $d_{i} \geq 0$.

Queremos verificar primeiramente que existe um ponto de equilíbrio $\bar{v}$ espacialmente constante .

Isto é equivalente a provar que existe $v$ tal que $Z(v)=0$. Temos os seguintes resultados: 
i) Se $A$ não é inversível, então existe $\omega \in K \operatorname{Ker} A$, que é uma solução do nosso problema.

ii) Se $A$ é inversível, seguindo os passos de Vickers [29], para nosso sistema de reação difusão, as $v_{i}$ não são mais frequências. Nestas condições temos que se $\frac{v}{V}=\omega, A \omega=\omega^{t} A \omega . \mathbb{I}$, então fazendo $A \omega=x$, temos que $w=A^{-1} \mathbb{I}$. $\left(\mathbb{I}^{t}\left(A^{-1}\right)^{t} \mathbb{I}\right)^{-1}$ é a solução que procuramos, onde $\mathbb{I}$ é o vetor coluna o qual todas suas entrada são iguais a um.

Prova:

i) Imediato.

ii) Basta verificar que $w=\mathbb{I}$. $\left(\mathbb{I}^{t}\left(A^{-1}\right)^{t} \mathbb{I}\right)^{-1}$ satistaz $A w=w^{t} A w \mathbb{I}$, de fato primeiramente temos que $A w=\frac{\mathbb{I}}{\left(\mathbb{I}^{t}\left(A^{-1}\right)^{t \mathbb{I}}\right)}$, por outro lado temos que, $w^{t} A w=\frac{\left(A^{-1} \mathbb{I}\right)^{t}}{\left(\mathbb{I}^{t}\left(A^{-1}\right)^{t} \mathbb{I}\right)} \cdot \frac{\mathbb{I}}{\left(\mathbb{I}^{t}\left(A^{-1}\right)^{t} \mathbb{I}\right)}=1$. Logo segue que $A w=w^{t} A w \mathbb{I}$. É fácil ver que $\sum \bar{m}_{i}=1$.

Nós estudaremos a estabilidade, sem trabalhar com a forma da solução, pois esta dependerá do tamanho assim como da forma do domínio $\Omega$. Usaremos os resultados de D. Henry expostos no capítulo 2 .

Pela equação (4) temos que os equilíbrios não são isolados, logo não se pode aplicar diretamente estes Teoremas na equação (6.3) pois esta não poderá ter um equilíbrio assintoticamente estável. Para contornar este problema, tentaremos identificar todos estes pontos da equação (6.3). A primeira idéia seria incorporar a condição: $\int_{\Omega} \sum v_{i}(x, 0) d x=\int_{\Omega} \sum \bar{v}_{i} d x$ no espaço, onde $\bar{v}$ é um equilíbrio espacialmente constante qualquer. Esta condição é invariante pois $V(x, t)$ é constante, mas não é linear. Então, seja $W^{\alpha} \subset X^{\alpha}$ definido por $W^{\alpha}=\left\{v \in X^{\alpha} ; \int_{\Omega} \sum v_{i}(x, 0) d x=\int_{\Omega} \sum \bar{v}_{i} d x\right\}$. Mas $W^{\alpha}$ com esta condição não é um espaço vetorial.

Para consertar isto, basta mudar de variável em (6.3) e fazer $w_{i}=v_{i}-\bar{v}_{i}$ no espaço $W^{\alpha}$ tal que $\sum w_{i}=0$, logo temos que nossa equação agora é

$$
\left\{\begin{array}{c}
\frac{\partial w_{i}}{\partial t}=z_{i}(w+\bar{v})+d_{i} \Delta w_{i} \quad 1 \leq i \leq r \\
\frac{\partial w_{i}}{\partial n}=0
\end{array}\right.
$$

onde $z_{i}(v)$ é dada por (6.2).

Lema 7.1.0.1 O espaço $W_{1}^{\alpha}=\left\{w \in X^{\alpha} ; \sum w_{i}=0\right\}$ é um espaço vetorial.

Lema 7.1.0.2 O equilibrio (agora zero) é isolado em (7.1). 


\section{Prova:}

Como o número de zeros da equação $z_{i}(w+\bar{v})=0$ é finito, pois não é mais homogênea, ( se multiplica mos $z_{i}$ por $V^{2}$, este será um polinômio) temos que qualquer outra raiz $\left(w^{\prime}\right)$ tem que satisfazer $\sum w_{i}^{\prime}=0$, mas isto significa que alguma componente $v_{i}^{\prime}-\bar{v}_{i} \neq 0$ portanto temos que $\left\|w^{\prime}-o\right\|>0$, portanto o (zero) é isolado.

Observação 7.1.1 Note que ainda temos a propriedade de dissipação pedida pois

$$
\frac{z_{i}(v)}{v_{i}-\bar{v}_{i}}=\frac{v_{i}}{v_{i}-\bar{v}_{i}}\left\{\frac{(A(v))_{i}}{\|v\|}-\frac{(v)^{t} A(v)}{\|v\|^{2}}\right\} \leq 2\|A\|_{1}
$$

O que estamos fazendo é trasladar o problema de $\bar{v}$ para a origem.

\subsubsection{Primeiro caso com fronteira Neumann}

Agora consideramos uma região $\Omega \subset R^{l}$ que é limitada e tem fronteira suave $\partial \Omega$, condição Neumann imposta em $\partial \Omega$.

Suponhamos que $m$ é um ponto de equilíbrio assintoticamente estável da equação (1), mas $m$ não é um E.S.S. (segundo definido na introdução). Então existe um vetor de probabilidade $q$ para o qual

$$
q^{t} A q \geq m^{t} A q
$$

isto é $m$ é invadível por $q$. O caso mais fácil a investigar é quando $q$ é uma estratégia pura, que no seguinte teorema escolheremos como sendo o vetor $(1,0,0, \ldots)$.

Teorema 7.1.1 Seja $m$ um ponto de equilíbrio temporalmente estável do sistema (1) para o qual $m_{i} \geq 0$ para todo i. Se

$$
a_{11} \geq\left(m^{t} A\right)_{1}
$$

então existe um conjunto de coeficientes de dispersão $d_{i}>0$, para todo $i$, tal que $m$ é um ponto assintoticamente instável da equação (3), isto é, se uma estratégia pura pode invadir $m$ então não é uniformemente espacialmente estável. 
Prova:

Desejamos aplicar os Teoremas (2.2.2) e o Teorema (5.1.2). Tendo em conta a observação anterior temos que mudar de variável em (1) e considerarmos o sistema

$$
\frac{\partial w_{i}}{\partial t}=z_{i}(w+\bar{v})+d_{i} \Delta w_{i}, \quad 1 \leq i \leq r
$$

Com isto estamos nas hipóteses do teorema pois $h=z_{\Omega}$ é Liptchitz e 0 é um ponto de equilíbrio isolado. Estudaremos a estabilidade do zero. Temos que a linearização em zero é a mesma que a de $z_{i}(v)$ em $\bar{v}$. Tendo em conta (8), seja $b_{i j}=m_{i} a_{i j}-m_{i}\left(m^{t} A\right)_{j}$ fazendo $1=d_{2}=d_{3}=d_{4} \ldots$. . A linearização em 0 tem espectro consistindo dos $\lambda$ tais que

$$
\operatorname{det}\left[b_{i j}+\left(d_{i} \rho-\lambda\right) \delta_{i j}\right]=0 \quad(* *)
$$

para algum $\rho \geq 0$. Então para $\rho$ suficientemente grande, o sinal do determinante estará determinado pelo sinal do $b_{11}+\left(d_{1} \rho-\lambda\right)$. Claramente podemos fazer este termo positivo ou negativo variando $d_{1}$ com o qual a equação $(* *)$ é satisfeita pelo conjunto dos $d_{i}$ e $\lambda$ positivos. Então $m$ é uma solução instável do sistema (3), pelo teorema (2.2.2).

Queremos provar o seguinte Teorema, que generaliza o Teorema (2.2) de [29].

Teorema 7.1.2 Seja m uma solução temporalmente estável do sistema (1) com todos os $m_{i}>0$, então $m$ é uniformemente estável.

\section{Prova:}

Tendo em conta as observações anteriores, temos que a linearização em 0 tem espectro consistindo $\operatorname{dos} \lambda$ tais que

$$
\operatorname{det}\left|-\left(d_{i} \rho+\lambda\right) I+D_{m} z\right|=0
$$

para algum $\rho \geq 0$. Portanto, o espectro é

$$
\left\{-\beta+d_{i} \rho \text { onde } \beta \text { é autovalor de } D_{m} z\right\} .
$$

Portanto, como $m$ é temporalmente estável do sistema (3) temos pelo teorema (2.2.1) (Henry) que $m$ é uniformemente espacialmente estável. 


\subsubsection{Segundo caso com controle pela fronteira}

Nesta seção queremos provar que se mudarmos o fluxo pela fronteira, obteremos os mesmos resultados de Vickers, sem impor que a estratégia seja ganhadora ou não, isto é queremos provar que se $\bar{m}$ é um ponto de equilíbrio da equação (6.4) então modificando a condição na fronteira de $\Omega$ temos um ponto de equilíbrio uniformemente espacialmente estável. Ou seja, se $\bar{m}$ é uma estratégia ganhadora (ou não) modificando o fluxo pela fronteira podemos torná-la uma estratégia ganhadora. Considere agora o sistema seguinte

$$
\left\{\begin{array}{c}
\frac{\partial \bar{u}_{i}}{\partial t}=d \Delta u_{i}+z_{i}(u-\bar{m}) \\
\frac{\partial \bar{u}_{i}}{\partial n}=-2 d_{0}\left(2-e^{-\|u-\bar{m}\|^{2}}\right)\left(u_{i}-m_{i}\right)
\end{array} \quad 1 \leq i \leq r,\right.
$$

Observação 7.1.2 O problema linearizado em $\bar{m}$ é

$$
\left\{\begin{array}{c}
\frac{\partial \bar{u}_{i}}{\partial t}=d_{i} \Delta u_{i}+\left(D_{\bar{m}} z\right)_{i}(u) \\
\frac{\partial \bar{u}_{i}}{\partial n}=-2 d_{0}\left(u_{i}\right)
\end{array} \quad 1 \leq i \leq r,\right.
$$

Pois

$$
\begin{aligned}
& \frac{\partial k_{i}}{\partial v_{i}}=-2 d_{0}\left(2-e^{-\|u-\bar{m}\|^{2}}\right)+4 d_{0}\left(2-e^{-\|u-\bar{m}\|^{2}}\right)\left(u_{i}-m_{i}\right)^{2} \\
& =-2 d_{0}\left(2-e^{-\|u-\bar{m}\|^{2}}\right)\left(2\left(u_{i}-m_{i}\right)^{2}+1\right) \\
& \frac{\partial k_{i}}{\partial v_{j}}=+4 d_{0}\left(2-e^{-\|u-\bar{m}\|^{2}}\right)\left(u_{i}-m_{i}\right)\left(u_{j}-m_{j}\right) .
\end{aligned}
$$

e portanto $\frac{\partial k_{i}}{\partial v_{j}}(m)=0$. Então fazendo uso da obsevação 6.2 , o problema linearizado é

$$
\left\{\begin{array}{c}
\frac{\partial u_{i}}{\partial t}=d \Delta u_{i}+D_{\bar{m}} z_{i} u \\
\frac{\partial u_{i}}{\partial n}=-2 d_{0}\left(u_{i}\right)
\end{array} \quad 1 \leq i \leq r\right.
$$

Teorema 7.1.3 Seja $m$ um ponto de equilibrio temporalmente estável do sistema (1), então existe uma função $k$ tal que $\bar{m}$ é um ponto uniformemente espacialmente estável da equação (6.4).

\section{Prova:}

O problema linearizado de (6.4) é equivalente ao problema (linearizando em $m=0$ )

$$
\left\{\begin{array}{c}
\frac{\partial u}{\partial t}=D \Delta u+\left(D_{0} z\right)(u) \\
\frac{\partial u}{\partial n}=\left(D_{0} k\right)(u)
\end{array}\right.
$$


o qual corresponde ao sistema da forma

$$
\left\{\begin{array}{c}
\frac{\partial u}{\partial t}=D \Delta u+B u \\
\frac{\partial u}{\partial n}=C u
\end{array}\right.
$$

onde $C_{i}=\left(D_{0} k\right)_{i}$ (a i-ésima linea da matriz $\left(D_{0} k\right)$ ) fazendo $v=e^{-B t} u$, temos que é equivalente ao

$$
\left\{\begin{array}{l}
\frac{\partial v}{\partial t}=D \Delta v \\
\frac{\partial v}{\partial n}=C v
\end{array}\right.
$$

logo, como $C$ é diagonal pela observação anterior, o sistema anterior é desacoplado portanto fazendo uso da observação do apêndice A.0.3 temos que o primeiro autovalor é positivo, então como $u=e^{(B+L) t} u_{0}$ é solução de (7.6), onde $L$ é o gerador infinitesimal definido pelo problema (7.6), e usando as estimativas da seção (4.6), temos o desejado. Logo o problema (6.4) tem zero como solução uniformemente espacialmente estável, pelo teorema (2.2.1).

O teorema seguinte generaliza o teorema anterior, mais ainda, vemos como, por meio da condição da fronteira, podemos controlar a instabilidade da equação (1), isto é, se $\bar{m}$ é uma estratégia instável ganhadora (ou não) de (1), impondo uma condição na fronteira apropriada podemos torná-la unifomemente espacialmente estável. Ou seja, que de novo por meio da condição imposta na fronteira podemos generalizar os resultados de [29] substituindo a condição de ser uma estratégia ganhadora pelo controle na fronteira.

Teorema 7.1.4 Seja $\vec{m}$ um ponto de equilibrio temporalmente instável do sistema (1), tal que $|\operatorname{Re} \sigma(D z(0))| \leq \varepsilon$. Então existe uma função $k$ tal que se $\varepsilon$ é suficientemente pequeno, $\bar{m}$ é um ponto uniformemente espacialmente estável da equação (3).

\section{Prova:}

Seguindo como na demostração anterior e pela observação anterior, temos a equação

$$
\left\{\begin{array}{c}
\frac{\partial v}{\partial t}=D \Delta v \\
\frac{\partial v}{\partial n}=C v
\end{array}\right.
$$

a qual gera um semigrupo $T_{D}(t)$. Usando as estimativas de Henry [11], temos que 


$$
\left\|e^{(B+S) t} u_{0}\right\| \leq M e^{(-\delta+\epsilon) t}\left\|u_{0}\right\|, \quad t \geq 0
$$

onde $S$ é o gerador infinitesimal do problema (7.7) logo fazendo uso da observação do apêndice A.0.3 podemos escolher $C$ de modo que o primeiro valor próprio do problema (7.7) seja positivo, portanto, se $\varepsilon$ é suficientemente pequeno, temos a estabilidade desejada. 


\section{Apêndice A}

\section{Autovalores e Parâmetros}

Neste capítulo estão reunidos alguns resultados encontrados em [21], [22] e [23]. Seja o operador diferencial definido pela expressão

$$
L^{c}[u]=\sum_{i, j=1}^{n} s_{i j}(x) \frac{\partial^{2} u}{\partial x_{i} \partial x_{j}}+\sum_{i=1}^{n} b_{i}(x) \frac{\partial u}{\partial x_{i}}-c u
$$

onde :

i) $x=\left(x_{1}, \ldots \ldots, x_{n}\right)$.

ii) $s_{i j}$ é simétrica e definida positiva.

iii) Todos os coeficientes são reais e limitados em $\Omega$.

Suponhamos que a fronteira $\partial \Omega$ esteja dividida em duas partes disjuntas $\Gamma_{1}$ e $\Gamma_{2}$ e as condições de fronteira são $u=0 \mathrm{em} \Gamma_{1} \mathrm{e}$

$$
M^{d}[u]=\sum_{i=1}^{n} e_{i}(x) \frac{\partial u}{\partial x_{i}}-d u=0 \quad \text { em } \quad \Gamma_{2}
$$

onde o campo de vetores $e$ aponta para fora de $\Omega$.

Consideremos o problema de autovalor

$$
(P)_{c, d}=\left\{\begin{array}{c}
L^{c}[u]-\lambda u=0 \quad \text { em } \quad \Omega \\
u=0 \quad \text { em } \quad \Gamma_{1} \\
M^{d}[u]=0 \quad \text { em } \quad \Gamma_{2}
\end{array}\right.
$$

Teorema A.0.5 (Protter, Weinberger [23]) Suponhamos que exista uma função $\omega$ satisfazendo as seguintes condições:
i) $\omega>0 \mathrm{em} \bar{\Omega}$
ii) $\omega \in C^{2}(\Omega) \cap C^{1}(\bar{\Omega})$ 
iii) $M^{d}[\omega] \geq 0 \quad$ em $\quad \Gamma_{2}$.

Então se o espectro do $P_{c, d}$ é não vazio, existe um número real $\lambda_{1}$ no espectro tal que o espectro está contido no semiplano

$$
\operatorname{Re} \lambda \geq \lambda_{1} \geq \inf \left(\frac{-L^{c}[\omega]}{\omega}\right)
$$

Lema A.0.2.1 Dados $d_{1}<d_{2}$, se $\lambda_{1}\left(\lambda_{2}\right)$ é o primeiro autovalor do $(P)_{0, d_{1}},\left((P)_{0, d_{2}}\right)$ então

$$
\lambda_{1}>\lambda_{2}
$$

Observação A.0.3 i)Para $c=0$ e $d=0$ temos que $\lambda_{1}=0$, portanto, $\lambda_{1}^{d}>0\left(\lambda_{1}^{d}<0\right)$ se $d<0,(d>0)$ e $c=0$.

ii)Para qualquer $d$ existe $c$ tal que $\lambda_{1}>0$. 


\section{Referências Bibliográficas}

[1] Alikakos, N.D., Regularity and Asymptotic Behavior for The Second Order Parabolic Equation with Nonlinear Boundary Conditions, Journal of Diff. Equat. 39 311-344 (1981).

[2] Amann, H., Semigroups and Nonlinear Evolution Equations, Linear Algebra and its Appl. 84, 3-32 (1986).

[3] Amann, H., Parabolic Evolution Equations and Nonlinear Boundary Conditions, Journal of Diff Eq. 72 201-269 (1988).

[4] Brezis, H., Análisis funcional Teoría y Aplicaciones, Alianza Editorial.

[5] Carvalho, A.N., Contracting Sets and Dissipation, Proccedings of the Royal Society of Edinburgh, 125A, 1305-1329, (1995).

[6] Carvalho, A.N., Oliva, S.M., Pereira, A.L. and Rodriguez - Bernal, A., Parabolic Problems with Nonlinear Boundary Conditions, Journal Math. Anal. Appl., 207, 409-461 (97).

[7] Fisher, R.A., The Wave of Advance of an Advantangeous Gene, Ann Eugen 7 355-369 (1937).

[8] Friedman, A., Partial Diferential Equations, Krieger 1964.

[9] Hale, J.K., Asymptotic Behavior of Dissipative Sistems, Mathematical Surveys and Monograph, 25, AMS, 1988.

[10] Hadeler, K., Diffusions in Fisher's Populations Model, Rocky Mt. J. Math. 11 39-45 (1981).

[11] Henry, D., Geometric Theory of Semilinear Parabolic Equations, Lecture Notes in Mathematics, 840, Springer-Verlag, 1981. 
[12] Henry, D., Some Infinite Dimensional Morse-Smale Systems Defined by Parabolic Diferential Equation, Journal of Diferential Equation 59, 165-205 (1985).

[13] Hutson, V.C.L., Vickers, G.T., Travelling wave and Domaninance of EES's, J.Math. Biol. 30, 457-471 (1992).

[14] Pao, C.V., Asymptotic Behavior and Nonexistence of Global Solutions for Class of Nonlinear Boundary Value Problems of Parabolic Type, Journal of Math. Anal. and Appl. 65, 616-637 (1978)

[15] Pazy, A., Semigrups of linear Operatorts and Applications to Parcial Diferencial Equations, Lecture Notes (1974)

[16] Ladyzenskaja, O.A and Uralceva, N.N., Equations aux derivées partialles de type elliptique, Monographies Universitaires de Mathématiques, Dunod Paris, (1968).

[17] Lions, J.L. and Magenes, E., Problemi ai Limiti non Omogenei V, Ann. Scuola Norm. Sup. Pisa 16, 1-44 (1962).

[18] Oliva, S.M, Pereira, A.L., Attractors for Parabolic Problems with Nonlinear Boundary Conditions in Fractional Powers Spaces, IME-USP Prepint Series \# 95-11 (1995).

[19] Maynard Smith, J., The Theory of Game and Evolution of Animal Conflicts. J. Theo. Biol. 47, 209-221 (1974)

[20] Maynard Smith, J., Price, G.T., The Logic of Animal Conflict. Nature, London. 246 15-18 (1973).

[21] Krein and Rutman, Linear Operators Leaving Invariant a Cone in a Banach Space, Amer. Math Soc. Trans. (1) 10, 3-95 (1962).

[22] Protter and Weinberger, Maximun Principles in Diff. Equations, Prentice-Hall, inc. (1967).

[23] Protter and Weinberger, On the Spectrum of General Second Order Operators, Bulletin Amer. Math. Soc., 72, 251-255,(1966).

[24] Seeley, R.,Norms and Domains of Complex Pawers $A_{B}^{z}$, Amer. J. Math. 93 299-309, (1971).

[25] Taylor, P., Jonker L., B., ESS and Game Dynamics, Math. Biosci. 40, 145-156 (1978). 
[26] Triebel, H., Interpoation theory, Funtion Spaces, Diferential Operators, North-Holland, Amsterdan, (1978).

[27] Vickers, G.T., Spatial Paterns and EES's, J. Theo. Biol 140, 129- 135 (1989).

[28] Vickers, G.T., Cannings, C. Patters of Ess's J. Theo. Biol. 132 387408 (1988).

[29] Vickers, G.T., Hutson, V.C.L., Budd, C.J., Spatial Paterns in Population Conflits. J. Math. Biol 31, 411-430 (1993).

[30] Zeeman, E.C., Global Theory of Dynamical Systems. In: Nitecki, Z., Robinson, C. (eds.) Proceedings of the Symposium of Northwestern University 1979. Berlin Heidelberg New York: Springer (1980). 